Article

\title{
Influence of the Framework Topology on the Reactivity of Chiral Pyrrolidine Units Inserted in Different Porous Organosilicas
}

\author{
Sebastián Llopis, Alexandra Velty and Urbano Díaz *(i) \\ Instituto de Tecnología Química, Universitat Politècnica de València-Consejo Superior de Investigaciones \\ Científicas, Avenida de los Naranjos s/n, E-46022 Valencia, Spain \\ * Correspondence: udiaz@itq.upv.es; Tel.: +34-963877811
}

Received: 14 June 2019; Accepted: 30 July 2019; Published: 31 July 2019

check for updates

\begin{abstract}
Three families of organosiliceous materials with different structuration level, order, and textural properties (non-ordered, M41S, and SBA-15 type materials) were prepared incorporating in their structural framework chiral pyrrolidine units with variable content. Likewise, non-ordered mesoporous hybrid solids were obtained through a sol-gel process in a fluoride medium, while M41S and SBA-15 type materials were obtained through micellar routes in the presence of long-chain neutral surfactants or block copolymers. Thanks to appropriate characterization studies and catalytic tests for the Michael addition between butyraldehyde and $\beta$-nitrostyrene, we showed how the void shapes and sizes present in the structure of hybrid materials control the diffusion of reactants and products, as well as confine transition states and reactive intermediates. The best catalytic results, considering activity and enantioselectivity, were achieved in the presence of a non-ordered material, NOH-Pyr-5\%, which exhibited the highest Brunauer-Emmett-Teller (BET) area, with a 96\% yield and a $82 \%$ ee for the Michael adduct.
\end{abstract}

Keywords: hybrid materials; heterogeneous catalysts; mesoporous organosilicas; chirality; Michael addition

\section{Introduction}

The combination in the same porous architecture of different units with several properties offers materials with characteristics that are not the simple sum of the properties of each individual unit, but provides materials with unique characteristics and reactivity [1,2]. This peculiarity becomes even more advantageous when the structural units present different natures, being combined at the nanometric scale to form new porous structures considered as hybrids [3,4]. In these cases, the reactivity exhibited by the hybrid materials is not only given by the isolated activity of the different active centers assembled in the structure, but also by the synergies and complementarity established between these materials $[5,6]$. These combined-active-centers systems are able to catalyze consecutive reactions with a high selectivity, using only an catalytic system that integrates all the adequate active centers $[7,8]$. In this type of solid, the existence of sub-domains in the structure with different marked hydrophobic or hydrophilic character will establish different concentrations of substrates and reaction intermediates due to differences in adsorption/desorption properties along the hybrid structural networks that contribute to a maximize yield and selectivity toward the desired reaction products [9].

The presence of different functional groups in the same structural porous framework can be achieved by using different structure units, organic and inorganic, which are assembled in a suitable way to be located in a homogeneous mode in the porous architecture in which they are integrated [10,11]. In particular, organic ligands, metallic nodes, or metal complexes, as well as 
organosilicon precursors (bridged silsesquioxanes) have been the structure units preferably used for the generation of porous hybrid materials at the nanometric scale [12,13]. The use of these units in solvothermal, hydrothermal, micellar, or sol-gel synthesis processes, with the occasional intervention of structure directing agents, facilitated the formation of new families of hybrid organic-inorganic materials $[14,15]$. These organic-inorganic architectures contain in their structure different active centers located in specific positions, such as metalorganic frameworks (MOFs) and periodic mesoporous organosilicas (PMOs) with different sorts of ordering, morphology, and porosity [16,17]. Special mention goes to solids obtained from disilanes that act as true molecular platforms that already contain in their initial conformation different functional groups present as a bridge, in the same organosilicon precursor, between two highly reactive terminal siloxane groups [18,19]. In these cases, it is possible to detect the presence of active centers separated by the controlled nanometric distance that avoid their mutual neutralization and maximize their reactivity and specificity when they are inserted in the final hybrid structure $[20,21]$. Organosiliceous mesoporous materials that contain different types of imidazole-type units in their structural framework are a clear example of this type of multifunctional solids [22]. Likewise, chiral centers, derived from cinchona, combined with other types of active groups that show a cooperative effect in the reactive process, have been observed in non-ordered porous organosilicas to carry out asymmetric multi-component reactions between aldehydes, malonates, and nitro compounds [23].

Despite the advantages associated with the presence of different active centers at the same time in their structure, which would make them optimal for their use in multi-stage catalytic processes, hybrid catalysts present certain aspects that still need to be overcome and is a research area currently in continuous expansion. One of their main weaknesses is the non-regular distribution of organic units in the structure that could reduce their catalytic performance [24]. This fact could be avoided with the use of organosilicon precursors, such as disilanes which already contained initially as a bridge the organic fragment with different active centers separated by controlled molecular distances. This approach would overcome the disadvantages that involve the use of several precursors simultaneously being involved in the synthesis process that favor the preparation of hybrid materials that are not homogeneous and exhibit a low crystallinity [25]. In this sense, the use of 1D organic-inorganic sub-units at the nanoscale that contain different active functions in their composition could be useful to prepare hybrid materials with different order levels and spatial dimensionalities with a regular and periodic distribution of active centers in their structure [26]. Some examples of 2D and 3D metal-organic hybrid materials have recently been prepared by following this methodology [27].

On the other hand, another aspect to consider would be to know the influence of the type and nature of the structural framework, in which the different active functions are included, over their final reactivity. This fact would be decisive to know the behavior of the same active center when it is incorporated in different organic-inorganic architectures, each of them with its characteristic morphology and porosity. Specifically, in the present work, three families of organosiliceous materials with different structuration level, order, and textural properties (non-ordered, M41S, and SBA-15 type materials) have been prepared, all of them having in common the presence of chiral pyrrolidine units in their structural framework with a variable concentration. Through appropriate characterization techniques, it has been possible to determine the different properties of the materials by performing catalytic tests that have been useful to determine how the reactivity and stereoselectivity of hybrid catalysts of a chiral nature are altered depending on the type of organic-inorganic structural framework in which the active functions are finally inserted.

\section{Results and Discussion}

\subsection{Synthesis and Characterization}

Herein we report the incorporation of pyrrolidine-type organic fragments into the structure of different mesoporous organosiliceous materials with different topologies and physico-chemical 
characteristics. The different properties of the synthesized hybrid materials determine the final reactivity of the organocatalytic moieties inserted and stabilized in their structure. The preparation of the hybrid materials begins with the synthesis of a bis-silylated monomer used as a precursor (PyrSil). This organosilicon precursor presents pyrrolidine-type fragments stabilized as organic bridges between the highly reactive terminal siloxane groups. Specifically, the monomer PyrSil was synthesized, starting from hydroxypyrrolidine (1), through successive reaction steps of protection with Cl-Bnz of pyrrolidine, reduction of ester group into hydroxyl (3) with $\mathrm{LiAlH}_{4}$, and a final condensation reaction with isocyanate silane molecules to form the silyl organosiloxane derivative (4). Therefore, the bis-silylated monomer, containing non-active pyrrolidine-urethane groups as organic bridges, were de-protected via reduction in the presence of a Pd/C catalyst under $\mathrm{H}_{2}$ to provide the final bridged silsesquioxane precursor (5), PyrSil, containing active functional pyrrolidine moieties in its composition (Scheme 1).

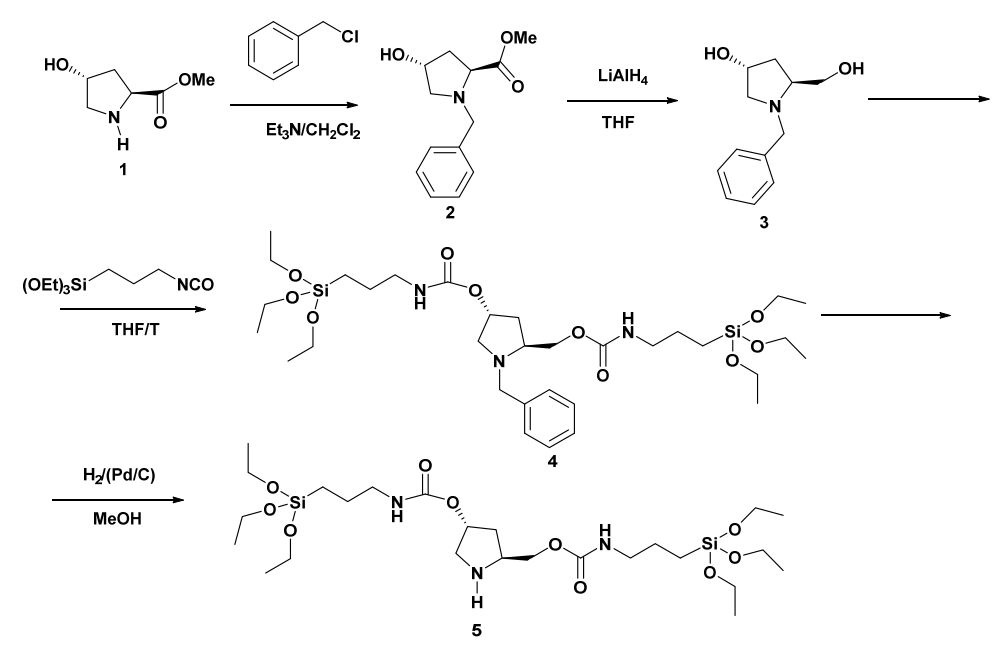

Scheme 1. Synthesis route to obtain the bis-silylated PyrSil silsesquioxane precursor.

Therefore, the bis-silylated monomer was used in different controlled hydrolysis and condensation synthesis processes, with and without structure-directing agents, to provide several families of organic-inorganic mesoporous materials. Specifically, different synthesis routes were explored to obtain ordered hybrid materials with MCM-41- and SBA-15-topology-types (M41S-Pyr and SBA-15-Pyr), depending on the use of long chain neutral amine surfactants or block copolymers during micellar processes together with the bis-silylated precursor. Non-ordered mesoporous hybrid solids were synthesized through a sol-gel process in a fluoride medium in the absence of structure-directing agents and under soft conditions at neutral $\mathrm{pH}$ conditions and at room temperature. In this case, the fluoride ions act as a mineralizing agent generating penta-coordinated organosilicon complexes as highly reactive intermediates that allowed the rapid gelation of the synthesis slurry. After the aging period at $36^{\circ} \mathrm{C}$, which allowed the assembly of the PyrSil monomers, the formation of non-ordered mesoporous structures (NOH-Pyr), containing in their framework active pyrrolidine units, was achieved (Scheme 2). 


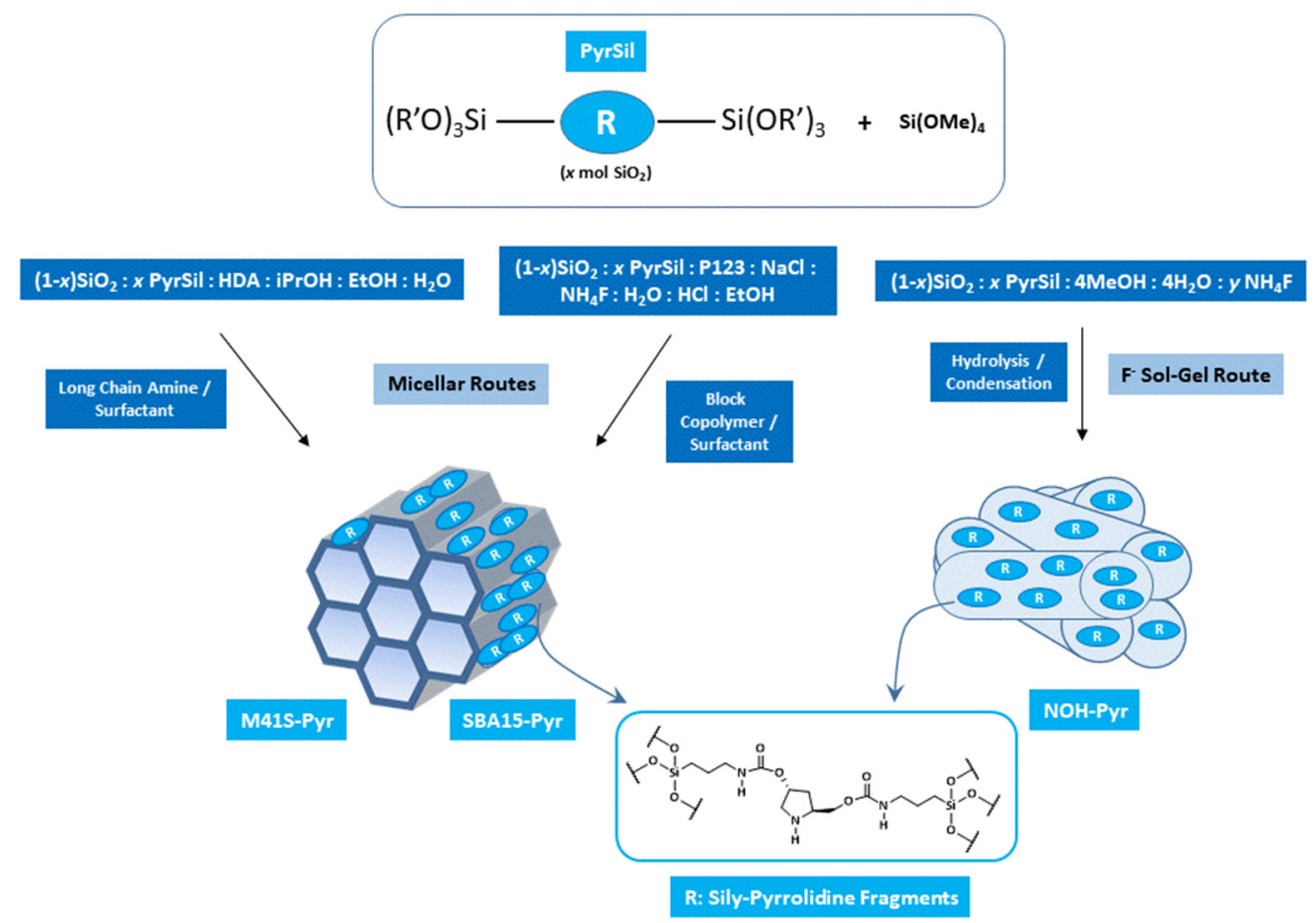

Scheme 2. Synthesis procedure of the different mesoporous hybrid materials through a sol-gel process in a fluoride medium to obtain non-ordered mesoporous chiral hybrid materials (NOH-Pyr) and micellar routes to obtain M41S-Pyr and SBA-15-Pyr materials using long-chain amines and block copolymers as structure-directing agents.

X-ray diffraction (XRD) patterns of ordered mesoporous materials prepared in the presence of structure-directing agents showed that the long-range order of the synthesized hybrid solids decreased significantly with the increase of the amount of pyrrolidine units incorporated in the structure. Likewise, the hybrid material incorporating a $30 \mathrm{~mol} \%$ of PyrSil in the synthesis gel did not present the diffraction band (100) characteristic of the hexagonal symmetry of the M41S-type materials prepared in the presence of long-chain surfactants. Similarly, the XRD pattern of solids prepared with block copolymers with high concentrations of pyrrolidine fragments did not display the low-angle diffraction bands characteristic of materials exhibiting a SBA-15-type structural arrangement. Nevertheless, in the specific case of the mesoporous hybrid solids obtained starting from $5 \mathrm{~mol} \%$ of the bis-silylated PyrSil precursor, it was observed that they reasonably conserved the low angle diffraction bands indicative of the M41S- and SBA-15-type ordering. For an amount of PyrSil precursor above $5 \mathrm{~mol} \%$ used in the synthesis procedure in the presence of structure directing agents, the progressive collapse of the mesoporous topology occurred due to the difficult assembling in an orderly manner of a high concentration of pyrrolidine fragments together with units of silicon tetrahedral along the walls of mesoporous channels (Figure 1). In the case of hybrid materials prepared through sol-gel processes in a fluoride medium in the absence of structure-directing agents, diffractograms (not shown) did not exhibit any diffraction bands as expected for mesoporous solids without any structural ordering. 

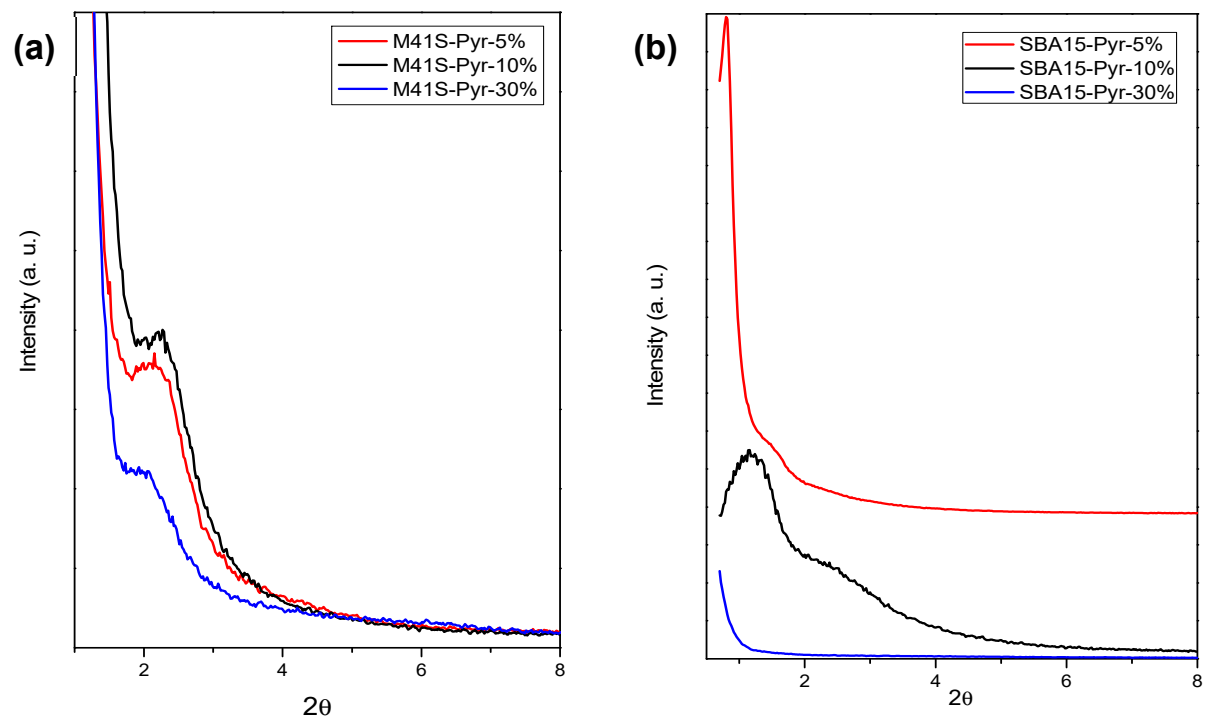

Figure 1. X-ray diffraction patterns of two different families of hybrid materials with various percentages of bis-silylated precursor, PyrSil, in the frameworks obtained after extraction processes to eliminate structure-directing agent molecules: (a) M41S-Pyr materials and (b) SBA-15-Pyr materials.
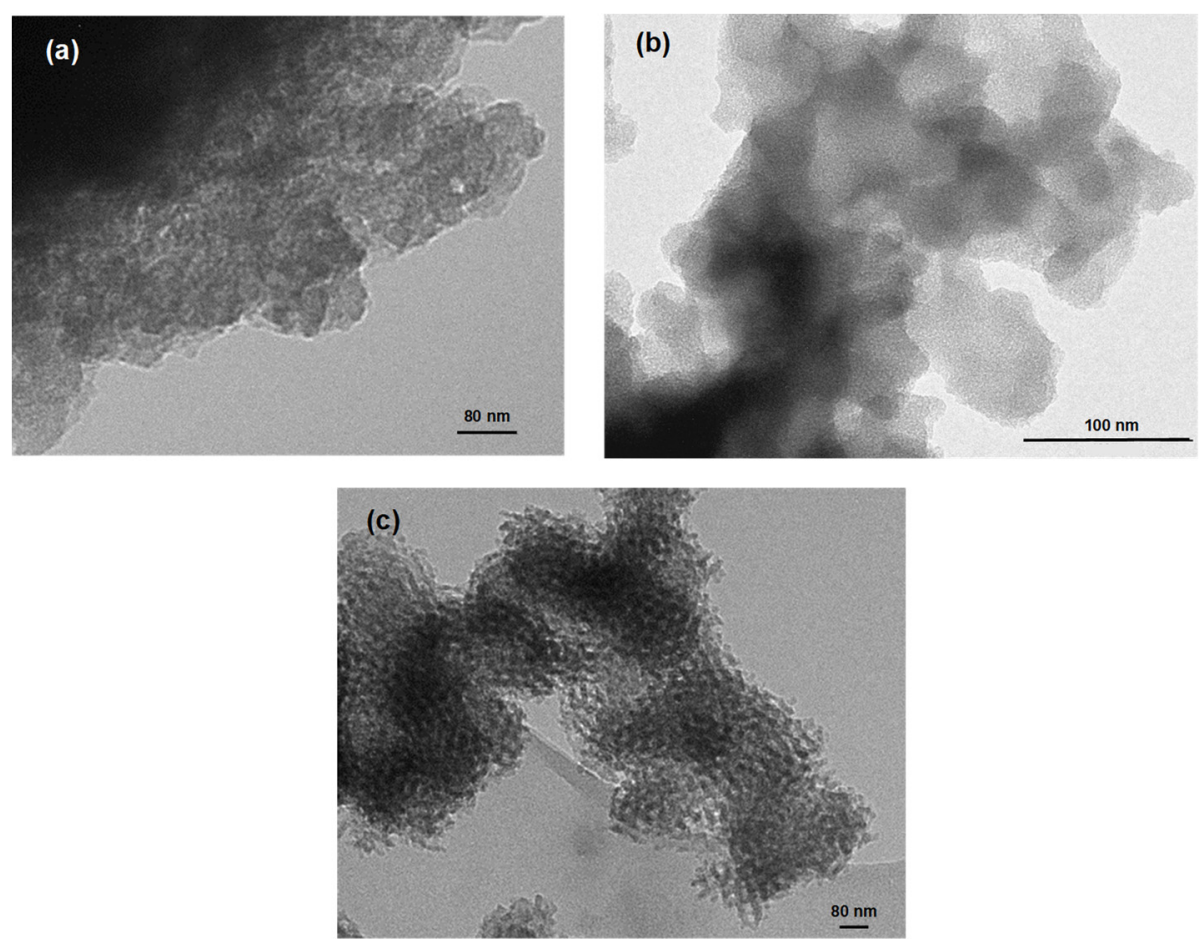

Figure 2. Transmission electron microscopy (TEM) micrographs of (a) NOH-Pyr-5\%, (b) M41S-Pyr-5\%, and (c) SBA-15-Pyr-5\%. Scale bars correspond to $80 \mathrm{~nm}$ for (a) and (c), and $100 \mathrm{~nm}$ for (b) images.

The images obtained using transmission electronic microscopy (TEM) showed how the mesoporous ordering of the hybrid materials was reasonably conserved in the samples that were prepared with up to $5 \mathrm{~mol} \%$ of PyrSil in the synthesis gel, although an elevated disorder and poor regular distribution of internal channels was observed. Specifically, in the ordered solids, cavities of approximately 30-100 were detected for materials with an SBA-15 or M41S topology, within the expected mesoporous range (Figure 2). In the non-ordered mesoporous solids, obtained through synthesis in a fluoride medium, exhibited an irregular morphology, where cavities of different diameters distributed in an irregular way were observed as expected. 
Through the chemical analysis (CHNS) of the final mesoporous samples, the increase of embedded organic content according to the amount of bis-silylated monomer (PyrSil) used in the synthesis process was confirmed. The as-synthesized ordered materials with topology SBA-15 and M41S were treated with different extraction methodologies in order to eliminate surfactant molecules and block copolymers that acted as structure-directing agents, with the aim of preserving the composition and structure of the pyrrolidine units inserted in the structural framework, and without carrying out a calcination process. However, although several successive extraction treatments were carried out, it was not possible to completely remove the molecules of the structure-directing agents. The maximum number of organic templates was eliminated when the content of pyrrolidine units was more elevated, with the elimination of structure directing agents in the samples obtained with $5 \mathrm{~mol} \%$ of PyrSil being more difficult. This matter could be associated with the poorer structuration level achieved for the samples with a higher content of organic pyrrolidine structural units in the framework that facilitated the easier elimination of internal template molecules. In the case of hybrid materials prepared in a fluoride medium in the absence of templates, all the organic content came from the units of pyrrolidine incorporated in the walls of the mesoporous solids. In fact, the calculated $\mathrm{C} / \mathrm{N}$ molar ratios fully coincided with the theoretical values $(\mathrm{C} / \mathrm{N}=4.3$ for a pyrrolidine-bridged unit) expected for the organic fragments from the PyrSil precursor, confirming that the organic units were preserved as they were initially after being incorporated into the mesoporous architectures (Table 1).

Table 1. Elemental analysis, $\mathrm{C} / \mathrm{N}$ molar ratios, and organic content estimated from the thermogravimetric (TGA) analysis for non-ordered, M41S, and SBA-15-type hybrid materials, as-synthesized and after the extraction process, containing different percentages of pyrrolidine moieties.

\begin{tabular}{llllll}
\hline Catalyst & \%C & \%N & C/N & \%TGA $^{\mathbf{a}}$ & \%E.A $^{\mathbf{b}}$ \\
\hline NOH-Pyr-5\% & 5.1 & 1.3 & 4.6 & 8.9 & 7.9 \\
NOH-Pyr-10\% & 9.4 & 2.4 & 4.5 & 16.4 & 13.9 \\
NOH-Pyr-30\% & 20.7 & 5.6 & 4.3 & 37.6 & 30.1 \\
M41S-Pyr-5\% as c & 21.7 & 2.6 & 9.9 & 45.9 & 28.6 \\
M41S-Pyr-5\% ext ${ }^{\text {d }}$ & 6.1 & 1.4 & 5.0 & 19.0 & 9.0 \\
M41S-Pyr-10\% as & 21.2 & 2.9 & 8.3 & 28.2 & 28.4 \\
M41S-Pyr-10\% ext & 10.8 & 2.6 & 4.8 & 19.0 & 15.7 \\
M41S-Pyr-30\% as & 36.2 & 5.0 & 8.4 & 52.4 & 49.6 \\
M41S-Pyr-30 ext & 21.2 & 5.2 & 4.8 & 41.9 & 30.3 \\
SBA-15-Pyr-5\% as & 21.0 & 1.2 & 20.6 & 27.3 & 25.7 \\
SBA-15-Pyr-5\% ext & 9.3 & 1.6 & 6.6 & 16.0 & 12.8 \\
SBA-15-Pyr-10\% as & 15.1 & 2.2 & 7.9 & 26.6 & 20.1 \\
SBA-15-Pyr-10\% ext & 10.6 & 2.6 & 4.8 & 18.6 & 15.2 \\
SBA-15-Pyr-30\% as & 22.1 & 5.5 & 4.6 & 45.3 & 31.5 \\
SBA-15-Pyr-30\% ext & 22.6 & 5.8 & 4.5 & 38.1 & 32.2 \\
\hline
\end{tabular}

${ }^{a}$ Organic content estimated from the thermogravimetric analysis; ${ }^{\mathrm{b}}$ Organic content estimated from CHNS elemental analysis; ${ }^{\mathrm{C}}$ as — synthesized hybrid materials; ${ }^{\mathrm{d}}$ ext-hybrid materials obtained after extraction processes to remove the structure-directing agents.

Figure 3 shows the thermogravimetric analysis (TGA) curves of the different organic-inorganic mesoporous samples obtained, as well as the corresponding derivatives (DTA) that allowed us to establish the thermal stability of the solids. The results showed two main weight losses. The first, located around $250-300^{\circ} \mathrm{C}$, was assigned to the remaining organic molecules of the structure-directing agents (surfactants or block copolymers (I)), which were not completely eliminated during the extraction process. Fragments of the silyl-propyl-pyrrolidine units inserted in the structure, coming from the PyrSil monomer precursor, could also be included in this first weight loss due to their decomposition during the thermal treatment. The second weight loss (II) was attributed to the included pyrrolidine fragments inserted into the structural frameworks. Specifically, this second weight loss was established in the temperature range of $300-500{ }^{\circ} \mathrm{C}$, providing evidence for the thermal stability of the solids obtained. In the case of the as-synthesized ordered mesoporous materials, before the extraction process, 
these two weight losses were better defined (see Supplementary Information). In all cases, an initial loss assigned to hydration water retained at low temperatures was observed, as well as a final loss (located around $550^{\circ} \mathrm{C}$ ) that was attributed to the hydroxylation water generated at high temperatures due to condensation phenomena existing between silanol groups present on the surface or in structure defects of the solids. In the case of the hybrid materials obtained without organic templates, only one weight loss was observed at $300-500{ }^{\circ} \mathrm{C}$ corresponding to silyl-pyrrolidine units present in the framework. It is noteworthy that the organic content was always higher when it was estimated using elemental analysis (CHNS) than when using thermogravimetric analysis (TGA) since in the latter, the oxygenated species contained in the hybrid materials are taken into account (Table 1).

(a)

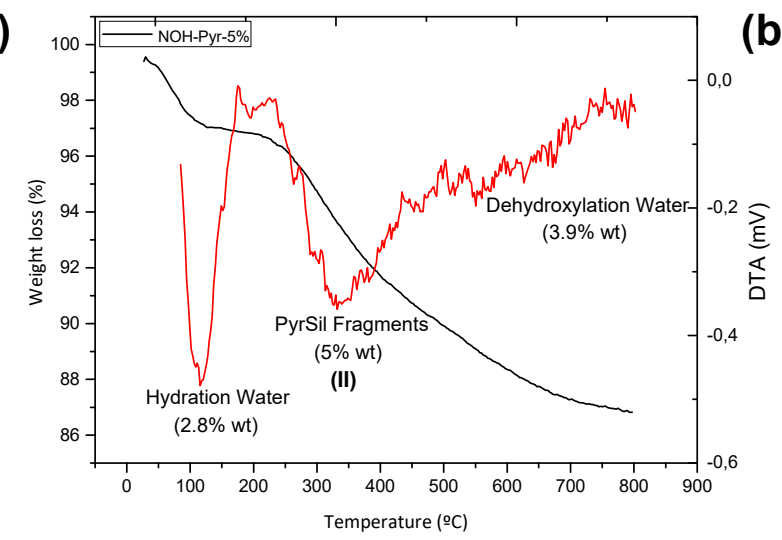

(b)

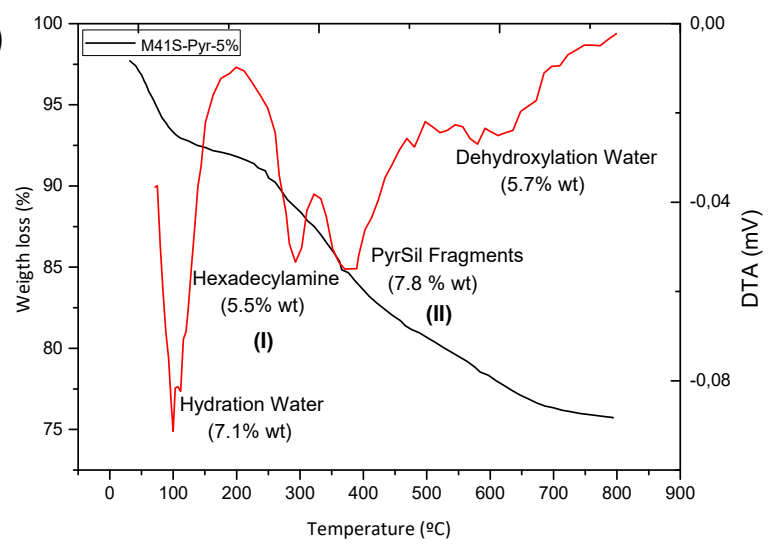

(c)

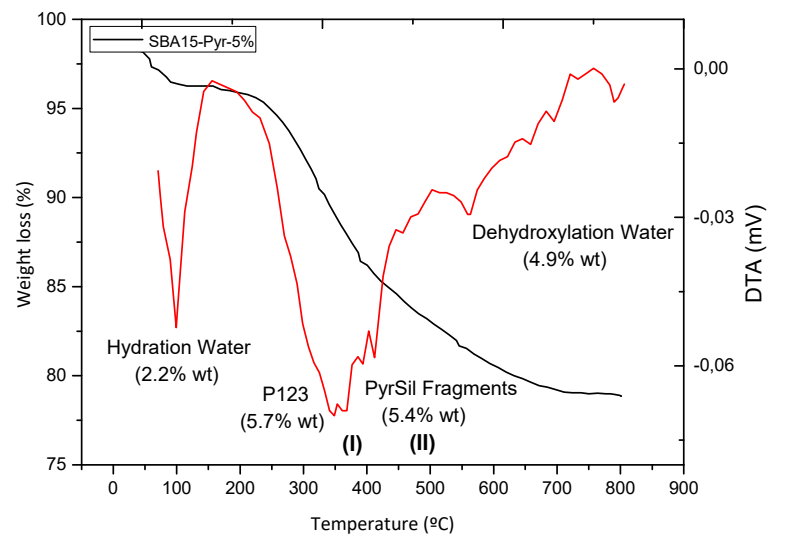

Figure 3. Thermogravimetrical curves (TGA) and corresponding derivatives (DTA) of (a) non-ordered materials (NOH-Pyr-5\%), (b) hybrid material M41S-type (M41S-Pyr-5\%), and (c) hybrid material SBA-15-type (SBA-15-Pyr-5\%).

Not only the presence, but also the integrity, of the pyrrolidine units inserted into the structure of the solids was confirmed through the results obtained using nuclear magnetic resonance (NMR) spectroscopy. Specifically, the ${ }^{13} \mathrm{C}$ NMR spectra showed, in all types of mesoporous hybrid materials, the chemical shifts, which were assigned to the carbon atoms from the bis-silylated precursor (PyrSil), including the atoms directly connected to the silicon atoms (Figure 4). These results corroborated the fact that the organic units present in the solids remained intact with the same composition as in the PyrSil precursor used during the synthesis process. However, in the extracted solids, some bands could be attributed to the rest of the structure-directing agent molecules (surfactants and block copolymers) that remained in the internal channels of the materials. 


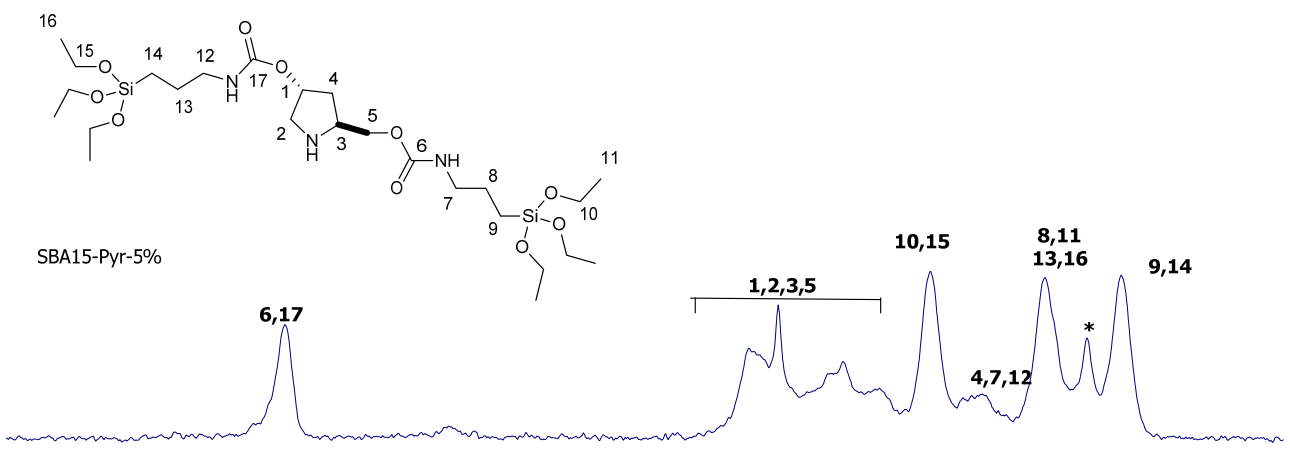

M41S-Pyr-5\%

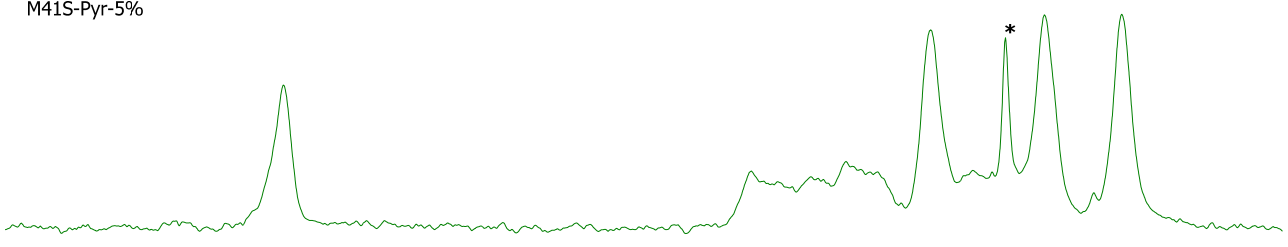

NOH-Pyr-5\%

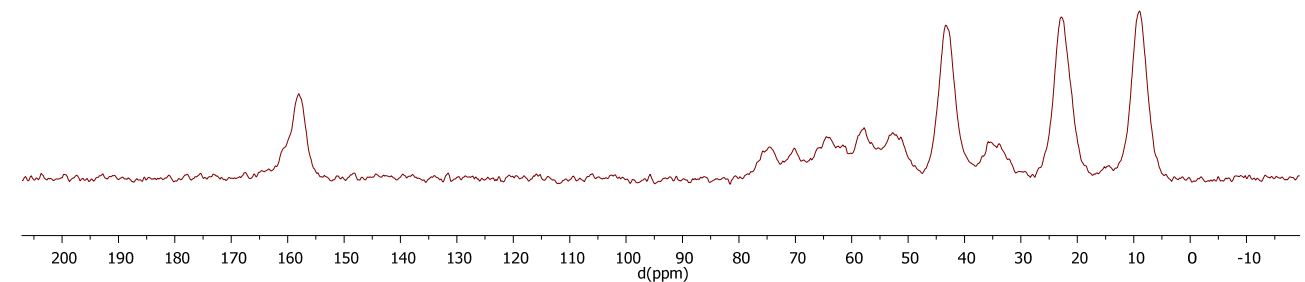

Figure 4. ${ }^{13} \mathrm{C}$ Magic Angle Spinning (MAS) NMR spectra of non-ordered and extracted M41S-type and SBA-15-type mesoporous hybrid materials containing pyrrolidine moieties and synthesized using 5 mol\% of PyrSil monomer precursor. Bands marked with an asterisk were assigned to chemical shifts due to rest of structure-directing agent molecules.

In addition, it was further confirmed through the ${ }^{29} \mathrm{Si} \mathrm{NMR}$ spectra that the active organic silyl-pyrrolidine fragments were actually covalently inserted into the walls of the porous materials. In all cases, the chemical shifts located in the range between -60 and $-80 \mathrm{ppm}$ were assigned to the T-type species of silicon atoms, such as $\mathrm{T}^{1}\left(\mathrm{C}-\mathrm{Si}(\mathrm{OH})_{2}(\mathrm{OSi})\right), \mathrm{T}^{2}\left(\mathrm{C}-\mathrm{Si}(\mathrm{OH})(\mathrm{OSi})_{2}\right)$, and $\mathrm{T}^{3}\left(\mathrm{C}-\mathrm{Si}(\mathrm{OSi})_{3}\right)$, were observed, together with the conventional Q-type silicon atom signals corresponding to the tetrahedral silicon units resulting from the condensation of tetramethyl-orthosilicate (TMOS) or tetraethyl-orthosilicate (TEOS) used in the synthesis processes. These results corroborated the fact that the propyl-silyl-pyrrolidine groups were covalently inserted into the structural framework of the solids obtained through an effective hydrolysis and condensation of the terminal siloxane groups of the bis-silylated precursor with the purely siliceous units (Figure 5). However, the presence of chemical shifts in ${ }^{13} \mathrm{C}$ NMR spectra assigned to terminal ethoxide groups present in the starting silyl-derivative precursors were indicative that the condensation phenomenon had only partially occurred (Figure 4). Furthermore, integrated values of chemical shifts corresponding to T-type silicon atoms corroborated that approximately 5,10 , and $30 \mathrm{~mol} \%$ of silyl-pyrrolidine units were effectively incorporated in the network through the different synthesis processes (see Table $\mathrm{S} 1$ and ${ }^{29} \mathrm{Si}$ Cross Polarization (CP)/MAS NMR spectra of hybrid materials in Supplementary Information). When comparing with the ${ }^{29}$ Si NMR spectrum of the monomer used as a precursor, it was observed that the chemical shifts of the T-type silicon atoms moved from a range of -40 to $-60 \mathrm{ppm}$ to -60 to $-80 \mathrm{ppm}$ that supported the successful integration of pyrrolidine fragments into the network of the solids (inset of Figure 5a). 

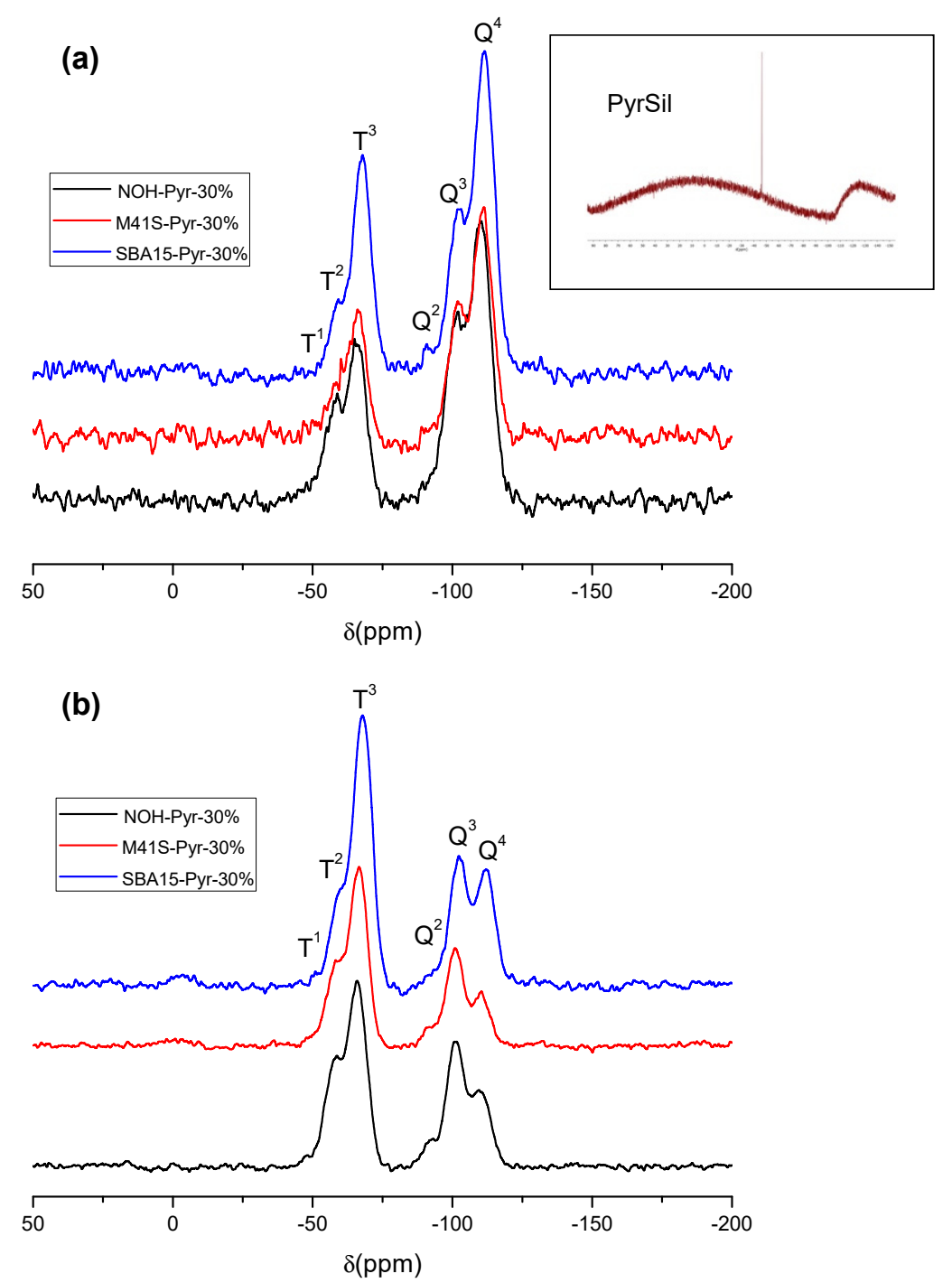

Figure 5. (a) ${ }^{29} \mathrm{Si} \mathrm{BD} / \mathrm{MAS}$ NMR spectra and (b) ${ }^{29} \mathrm{Si} \mathrm{CP} / \mathrm{MAS}$ NMR spectra of mesoporous hybrid materials (non-ordered and extracted M41S and SBA-15-type) with the highest percentage of pyrrolidine units included in the framework and the assignment of T- and Q-type silicon atoms.

Infrared spectroscopy was also useful to confirm the presence and integrity of the pyrrolidine units inserted covalently in the structure of the different types of prepared mesoporous hybrid materials (Figure 6). In all cases, the bands associated with the stretching vibrations were observed in the Fourier-transform infrared (FTIR) spectra $\left(v(-\mathrm{NH}-)\right.$ : shoulder at $\left.2620 \mathrm{~cm}^{-1}\right)$ and bending vibrations $\left(\delta(-\mathrm{NH}-): 1559\right.$ and $\left.1643 \mathrm{~cm}^{-1}\right)$ of the secondary amines present in the organic bridges included in the bis-silylated precursor (PyrSil) used in the synthesis processes, also including the cyclic aliphatic amines directly present in the pyrrolidine units. In addition, bands due to stretching vibrations attributed to the carbamate groups, $v(-\mathrm{NH}-\mathrm{CO}-\mathrm{O}-)$, were also detected at $1703 \mathrm{~cm}^{-1}$. The $-\mathrm{CH}_{2}$ - units included in the propyl chains located in the PyrSil precursor were observed through the existence of symmetric and asymmetric vibration bands, characteristic of alkylic groups linked to amino groups $\left(\mathrm{v}\left(-\mathrm{N}-\mathrm{CH}_{2}-\right)\right.$ : 2897 and $2958 \mathrm{~cm}^{-1} ; \delta\left(-\mathrm{N}-\mathrm{CH}_{2}-\right)$ : 1383 and $\left.1447 \mathrm{~cm}^{-1}\right)$. In this same range, vibrations due to the rest of the surfactant or block copolymer molecules that remained in the mesoporous channels, after the extraction processes, were also observed at 1500 and $1450 \mathrm{~cm}^{-1}, \delta\left(-\mathrm{N}-\mathrm{CH}_{2}-\right)$, corresponding to the presence of hexadecylamine and P123 block copolymers in M41S- and SBA-15-type hybrid materials, respectively. Specifically, we observed the signals due to $\mathrm{P} 123$ at 2880 and $2835 \mathrm{~cm}^{-1}$, characteristic of alkyl groups bonded to ether groups, $v\left(\mathrm{O}-\mathrm{CH}_{2}-\right)$, and bending vibrations, $\delta\left(-\mathrm{CH}_{3}-\right)$, at 1395 and 
$1365 \mathrm{~cm}^{-1}$. However, these vibrations were practically nonexistent, confirming the high effectiveness of extraction treatments (see the FTIR spectra in Supplementary Information, Figure S5). Additionally, a wide band centered at $3647 \mathrm{~cm}^{-1}$ was detected in all spectra associated with the surface silanol groups $(\mathrm{Si}-\mathrm{OH})$ that are usually found in organic silicates with a high density of structure defects. The vibration band observed at $950 \mathrm{~cm}^{-1}$ was also due to external silanols.

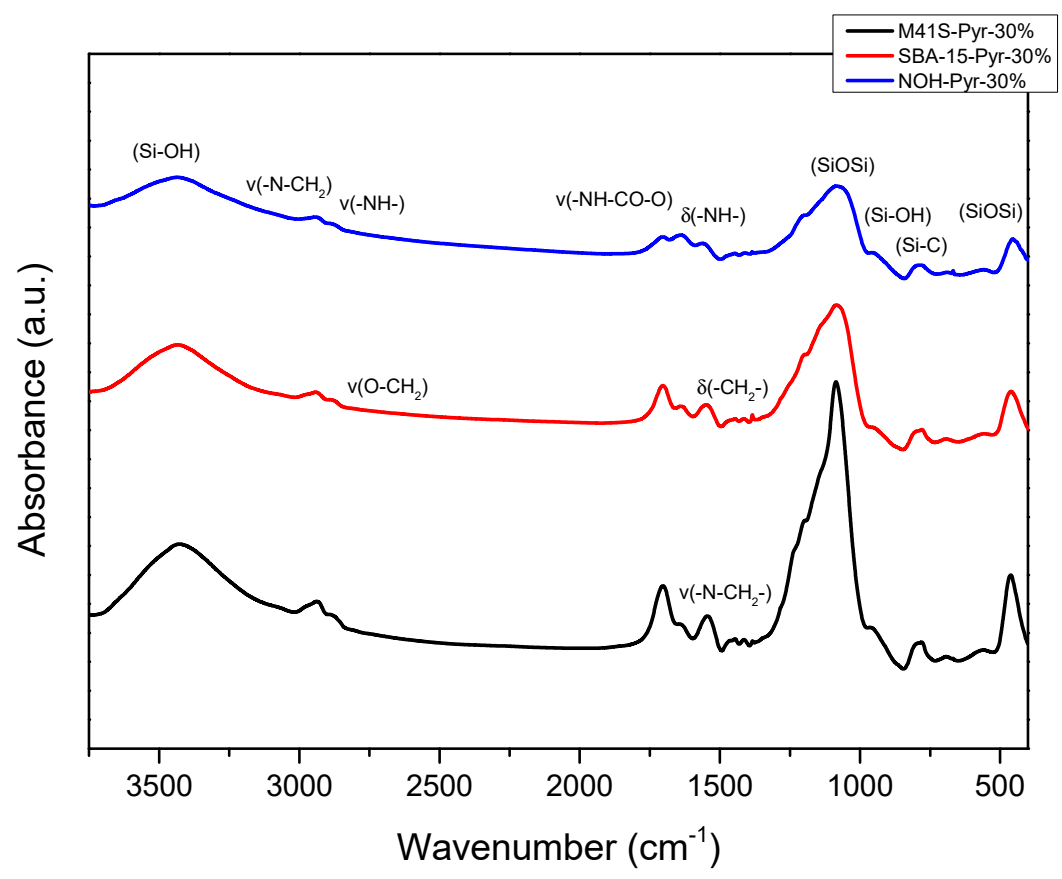

Figure 6. FTIR spectra of mesoporous materials (NOH-Pyr and extracted M41S- and SBA-15-types) with the highest pyrrolidine moieties content in the framework.

In the range of the infrared spectra corresponding to the vibrations characteristic of the structure framework, the band of the $\mathrm{Si}-\mathrm{C}$ vibration located at $790 \mathrm{~cm}^{-1}$ was observed, confirming the existence of bonds with a covalent nature established between the tetrahedral $\mathrm{SiO}_{4}$ units and the bis-silylated fragments containing pyrrolidine groups. Within this range of vibration, the conventional bands of the Si-O-Si groups (458 and $1080 \mathrm{~cm}^{-1}$ ) were also observed, which were the main structural components of the prepared organosiliceous hybrid materials, regardless of the achieved structural ordering level (Figure 6). Therefore, spectroscopic results (NMR and IR) clearly showed both the integrity of the pyrrolidine units and their effective incorporation through covalent bonds in the structure of the different porous hybrid materials.

The textural properties of the different hybrid materials (specific surface area and free porous volume) were studied using the nitrogen adsorption isotherms (Figure 7). The results showed how the materials obtained in the fluoride medium, without structure-directing agents, exhibited characteristic isotherms of non-ordered porous solids with a marked change of slope at high relative pressures $\left(\mathrm{P} / \mathrm{P}_{0} \approx 0.3-0.4\right)$, due to the existence of high-diameter pores in the internal mesoporous channels existing in the organic-inorganic framework. In this family of materials, the Brunauer-Emmett-Teller (BET) surface and total volume decreased as the concentration of pyrrolidine units inserted in its structure increased due to the greater difficulty in assembly between the several structure units when the number of organic fragments was more elevated. This fact caused the collapsing of the structure for the materials prepared with $30 \mathrm{~mol} \%$ of PyrSil as an initial precursor. In the case of non-ordered hybrid materials, the BET, surface, and the porous volume ranged from $636 \mathrm{~m}^{2} / \mathrm{g}$ to $5 \mathrm{~m}^{2} / \mathrm{g}$ when the amount of silyl-pyrrolidine units oscillated from $5-30 \mathrm{~mol} \%$ of total silicon, respectively (Table 2). 
Table 2. Textural properties of the hybrid mesoporous materials with different topology (non-ordered, M41S-, and SBA-15-type) and percentage of pyrrolidine fragments included in the framework.

\begin{tabular}{|c|c|c|c|c|c|c|c|}
\hline Catalysts & $\begin{array}{c}S_{\text {BET }} \\
\left.\left(\mathrm{m}^{2} / \mathrm{g}\right)\right)\end{array}$ & $\begin{array}{l}S_{\text {ext }^{a}} \\
\left(\mathrm{~m}^{2} / g\right)\end{array}$ & $\begin{array}{l}S_{\text {Micro }} \\
\left(\mathrm{m}^{2} / \mathrm{g}\right)\end{array}$ & $\begin{array}{c}V_{\text {tot }} \\
\left(\mathrm{cm}^{3} / \mathrm{g}\right)\end{array}$ & $\begin{array}{l}V_{\text {Micro }} \\
\left(\mathrm{cm}^{3} / \mathrm{g}\right)\end{array}$ & $\begin{array}{c}V_{\text {BJH }} \\
\left(\mathrm{cm}^{3} / \mathrm{g}\right)\end{array}$ & $\varnothing_{\text {Pore }}(\AA ̊)$ \\
\hline NOH-Pyr-5\% & 636 & 411 & 225 & 0.46 & 0.12 & 0.34 & 36 \\
\hline NOH-Pyr -10\% & 374 & 329 & 44 & 0.25 & 0.02 & 0.12 & 34 \\
\hline NOH-Pyr-30\% & 5 & 5 & 0 & 0.02 & 0 & 0.01 & n.a \\
\hline Pure Silica non-ordered & 668 & 547 & 121 & 0.52 & 0.05 & 0.47 & $20-30$ \\
\hline M41S-Pyr-5\% & 242 & 242 & 0 & 0.26 & 0.01 & 0.13 & 46 \\
\hline M41S-Pyr -10\% & 317 & 317 & 0 & 0.41 & 0 & 0.16 & 42 \\
\hline M41S-Pyr -30\% & 160 & 160 & 0 & 0.28 & 0 & 0.10 & 39 \\
\hline Pure silica M41S & 1149 & 1149 & 0 & 0.94 & 0 & 0.55 & 30 \\
\hline SBA-15-Pyr-5\% & 285 & 285 & 0 & 0.81 & 0 & 0.48 & 77 \\
\hline SBA-15-Pyr-10\% & 282 & 282 & 0 & 0.73 & 0 & 0.39 & 75 \\
\hline SBA-15-Pyr-30\% & 125 & 125 & 0 & 0.55 & 0 & 0.28 & 113 \\
\hline Pure silica SBA-15 & 870 & 747 & 123 & 1.28 & 0.06 & 1.05 & 61 \\
\hline
\end{tabular}

${ }^{\text {a }}$ From t-plot; $\mathrm{S}_{\mathrm{ext}}=\mathrm{S}_{\mathrm{BET}}-\mathrm{S}_{\text {Micro; }}$ BJH-Barrett-Joyner-Halenda Method

In contrast, organic-inorganic solids prepared in the presence of structure-directing agents (surfactants and block copolymers) exhibited the characteristic type IV isotherms of ordered mesoporous materials overall in the solids with a SBA-15 topology more than M41S-type materials. These isotherms showed a marked change of slope at relative pressures $\mathrm{P} / \mathrm{P}_{0}$ between approximately $0.5-0.6$, indicating the existence of internal channels with diameters within the range of the mesopore. However, this change of slope in the isotherms was attenuated in the M41S family showed a marked loss of structural order in comparison with SBA-15-type materials at the same level of pyrrolidine incorporation, which was corroborated using X-ray diffraction (Figure 1). As it happens in the case of non-ordered materials, it was observed that the specific surface area and the pore volume decreased markedly as the concentration of inserted pyrrolidine groups was higher, up to the point of collapse in the solids obtained by using $30 \mathrm{~mol} \%$ of PyrSil as the initial bis-silylated precursor during the synthesis process. In particular, the BET surface area for M41S- and SBA-15-type hybrid materials oscillated between $160-317 \mathrm{~m}^{2} / \mathrm{g}$ and $125-285 \mathrm{~m}^{2} / \mathrm{g}$, respectively. In both cases, the materials with the highest presence of silyl-pyrrolidine fragments exhibited the lowest BET surface due to the difficulties in assembling organic and inorganic units through hydrolysis and condensation processes. It is remarkable that solids obtained in the presence of $5 \mathrm{~mol} \%$ of PyrSil as a monomer precursor showed BET surfaces areas similar to those obtained with $10 \mathrm{~mol} \%$ of PyrSil. Additionally, we can observe that the materials with the highest percentage of PyrSil fragments showed BET surfaces higher than non-ordered materials, probably due to the better-achieved organization due to the presence of structure-directing agents. The results evidenced how the presence of pyrrolidine fragments in the framework strongly influenced the final textural properties of the porous hybrid solids.

From the distribution of the Barrett-Joyner-Halenda (BJH) pore diameter, it was observed that the internal pores of the different materials exhibited diameters between 75-110 ̊ for SBA-15-type and 30-40 A for M41S-type and non-ordered hybrid materials such as it was expected for this type of mesoporous solids, with the broadest pore size distribution for M41S-type materials. In the three families, it was observed that the pore sizes obtained were slightly higher than in the equivalent pure silica solids, indicating the inclusion of voluminous silyl-pyrrolidine fragments into the framework to obtain internal pores with higher diameters. 

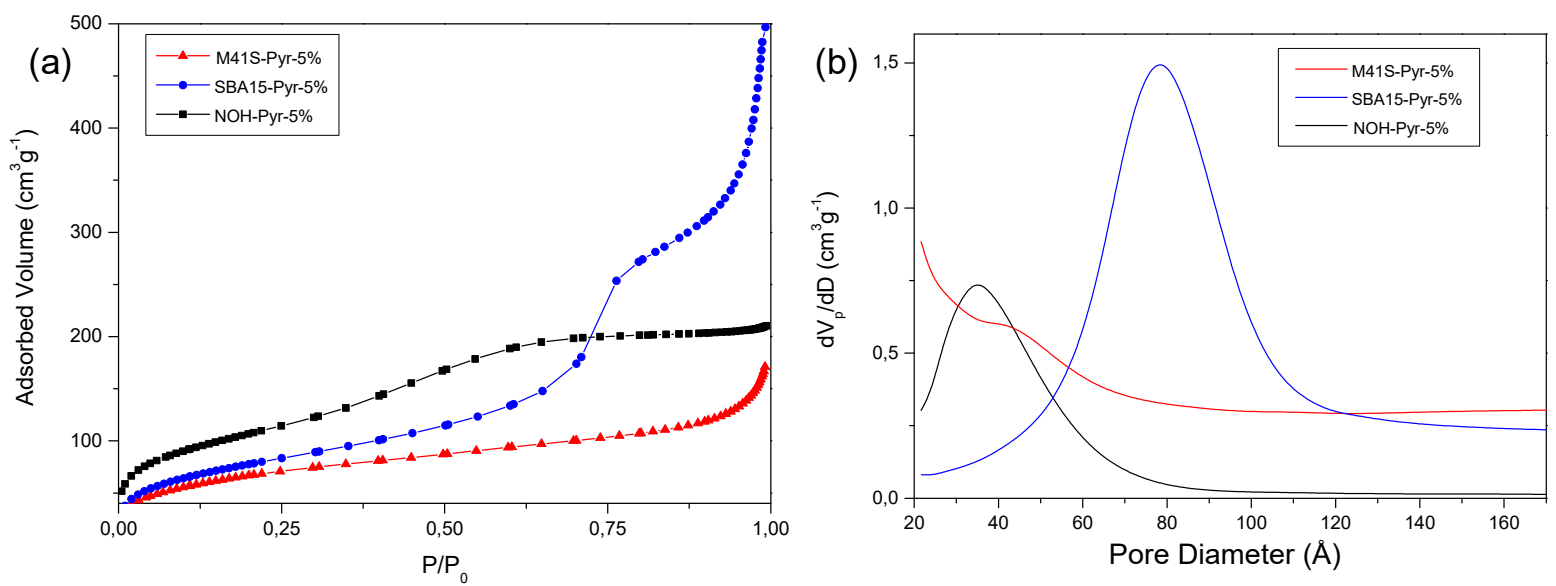

Figure 7. (a) $\mathrm{N}_{2}$ adsorption isotherms and (b) pore size distribution calculated using the BJH method of all materials containing $5 \mathrm{~mol} \%$ of PyrSyl fragments in the framework.

\subsection{Catalysis}

We explore the catalytic performance of the three families of organosiliceous materials with different structuration level, order, and textural properties in the asymmetric Michael addition of butyraldehyde and $\beta$-nytrostyrene. Recently, this catalytic process was successfully performed using amino acids adsorbed on inorganic oxides as laponite-type supports that formed active hybrid organic-inorganic chiral heterogeneous catalysts [28]. The preparation of carbonyl and nitrocarbonyl compounds through the asymmetric Michael addition reaction of carbonyl compounds and nitroolefins is a versatile and useful tool in organic synthesis, normally being carried out using homogeneous and heterogeneous catalytic systems containing prolinol active units [29-32]. The reaction mechanism widely accepted involves, first, the formation of the enamine via the condensation of a chiral amine and a carbonyl substrate. The following enamine addition onto the nitro alkene compound generates an iminium intermediate. The hydrolysis of the iminium delivers the Michael product with the regeneration of the initial asymmetric amine catalyst. The geometry of enamine is determined by the catalyst architecture, and E-enamine was thermodynamically preferred. Moreover, the chiral substituent on the catalytic solid influences the facial selectivity of the Michael addition via steric shielding and/or electronic interaction. For the Michael addition mechanism, the less-hindered SiSi transition state through an anti-enamine was usually favored for aldehydes, while the ReRe transition state through syn-enamine was usually preferred for ketones. Considering all-these mechanistic insights and the enantioselectivity reaction product, we previously reported a suggested mechanism of chiral amine-catalyzed Michael addition through E-enamine formation in the presence of pyrrolidine-carbamate units embedded in the framework (Scheme 3) [33]. 


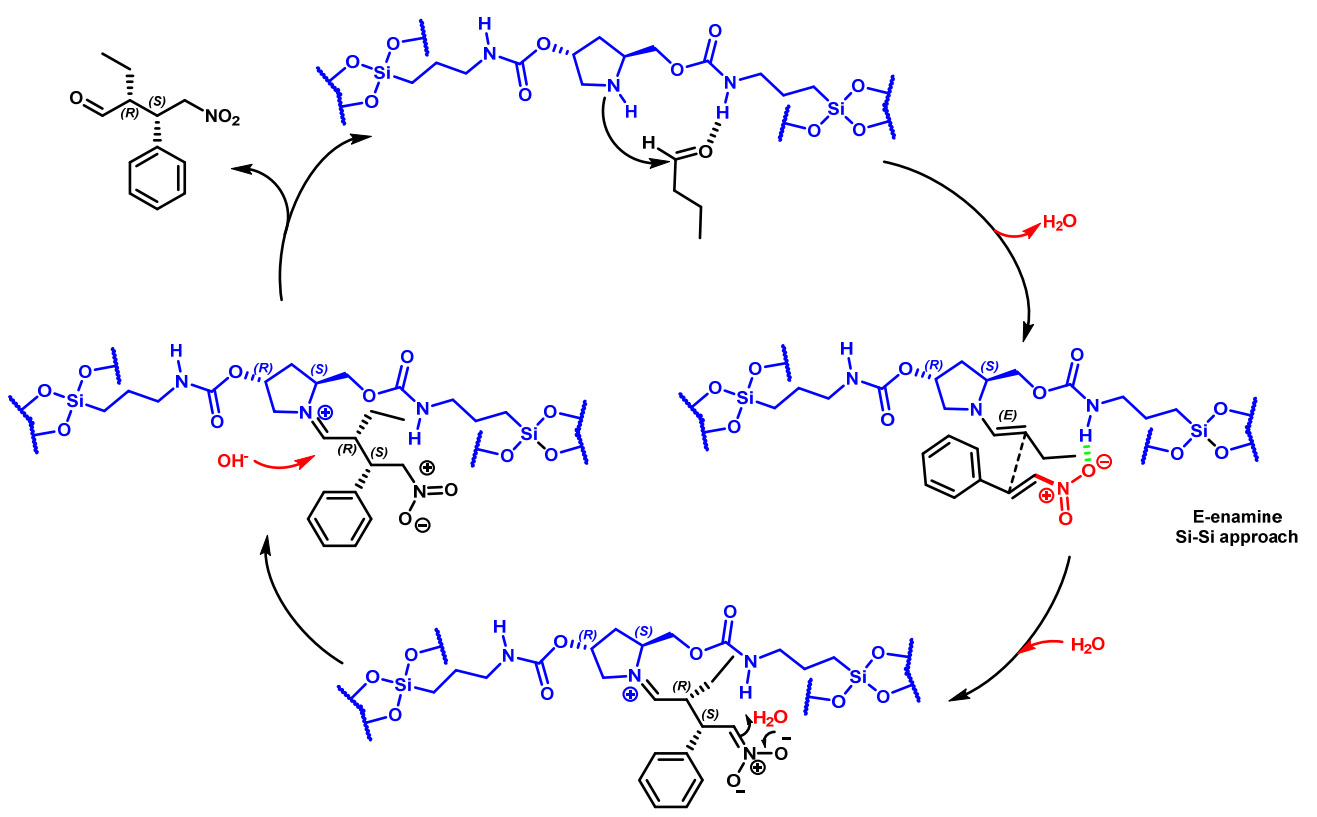

Scheme 3. Suggested mechanism of chiral amine-catalyzed Michael addition through E-enamine formation.

The catalytic behavior of the three families of materials with different contents of PyrSil and topology for the chiral Michael addition between butyraldehyde and $\beta$-nitrostyrene (Scheme 4) was studied in optimized reaction conditions, $20 \mathrm{~mol} \%$ of chiral catalyst at $15{ }^{\circ} \mathrm{C}$ in dichloromethane (DCM) [33]. In all cases, the (2R,3S)-2-methyl-4-nitro-3-phenylbutanal was the main isomer (syn).

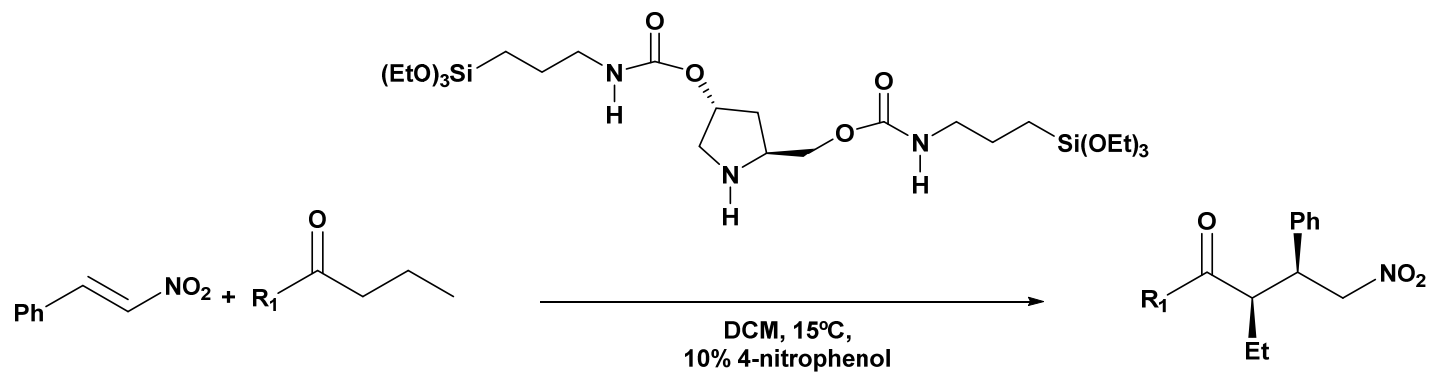

Scheme 4. Chiral amine included into mesoporous materials catalyzes the Michael addition between butyraldehyde and $\beta$-nitrostyrene.

From the nitrogen adsorption isotherms, it was concluded that materials obtained in a fluoride medium without structure-directing agents exhibited isotherms of non-ordered porous materials with high-diameter pores centered at $40 \AA$. Moreover, it was observed that the BET surface and total volume decreased with an increase of included pyrrolidine units in the structure producing the structural collapse at $30 \mathrm{~mol} \%$ of PyrSil. The catalytic results reported in Table 3 and Figure 8 show that the catalytic activity of the non-ordered porous materials clearly decayed with an increase of PyrSil content due the decrease of BET area, producing diffusion constraints and tended to zero with the collapse of the structure. Likewise, the best results in terms of activity and enantioselectivity were achieved with the NOH-Pyr-5\% catalyst, which presented the highest BET area (Table 3, entry 1), providing a (2R,3S)-2-methyl-4-nitro-3-phenylbutanal yield of $96 \%$ and an enantiomeric excess (ee) of $82 \%$. 
Table 3. Catalytic results achieved in the presence of the different hybrid materials for the enantioselective Michael addition.

\begin{tabular}{|c|c|c|c|c|c|c|c|}
\hline Entry & Catalyst & $\mathrm{r}_{0}\left(\mathrm{~mol} \cdot \mathrm{L}^{-1} \cdot \mathrm{h}^{-1}\right)$ & $\mathrm{K}\left(\mathrm{L} \cdot \mathrm{mol}^{-1} \cdot \mathrm{h}^{-1}\right)$ & $t(h)$ & Conv. (\%) & ee $\%$ & dr \\
\hline 1 & M41S-Pyr-5\% & 0.0196 & 0.2357 & 11 & 94 & 76 & $87: 13$ \\
\hline 2 & M41S-Pyr-10\% & 0.0139 & 0.167 & 15 & 95 & 74 & $88: 12$ \\
\hline 3 & M41S-Pyr-30\% & 0.0012 & 0.0141 & 96 & 35 & 66 & $88: 12$ \\
\hline 4 & SBA-15-Pyr-5\% & 0.0079 & 0.0945 & 31 & 93 & 76 & $90: 10$ \\
\hline 5 & SBA-15-Pyr-10\% & 0.0197 & 0.2365 & 15 & 99 & 76 & $89: 11$ \\
\hline 6 & SBA-15-Pyr-30\% & 0.0041 & 0.0491 & 45 & 93 & 68 & $88: 12$ \\
\hline 7 & NOH-Pyr-5\% & 0.0418 & 0.5013 & 10 & 96 & 82 & $92: 8$ \\
\hline 8 & NOH-Pyr - $10 \%$ & 0.0087 & 0.1048 & 38 & 86 & 72 & $88: 12$ \\
\hline 9 & NOH-Pyr -30\% & - & - & $\mathrm{n} / \mathrm{d}$ & - & - & - \\
\hline
\end{tabular}

Reaction conditions: $\beta$-nitrostyrene $(0.1 \mathrm{mmol})$, butyraldehyde $(1 \mathrm{mmol}), 10 \%$ 4-nitrophenol, $1 \mathrm{~mL}$ of DCM, and $20 \mathrm{~mol} \%$ of catalyst; ee: enantiomeric excess; dr: diastereoisomeric ratio. $\mathrm{r}_{0}$ : initial rate. K: kinetic constant.

(a)

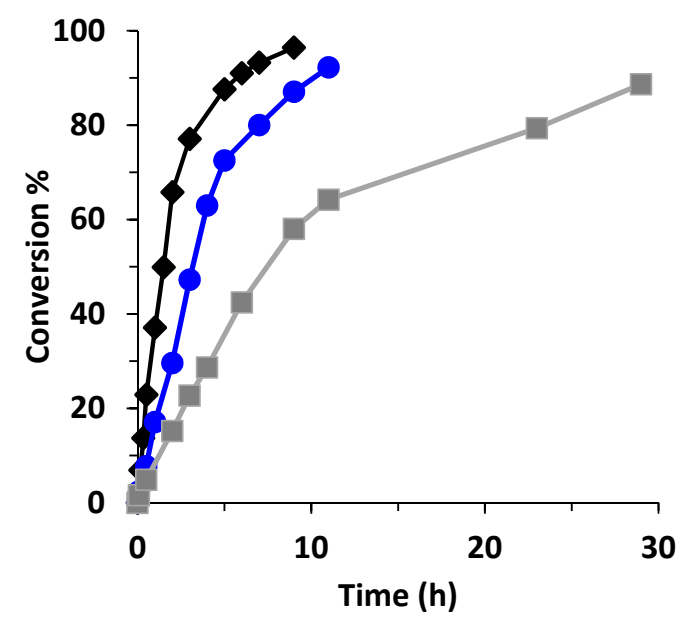

(b)

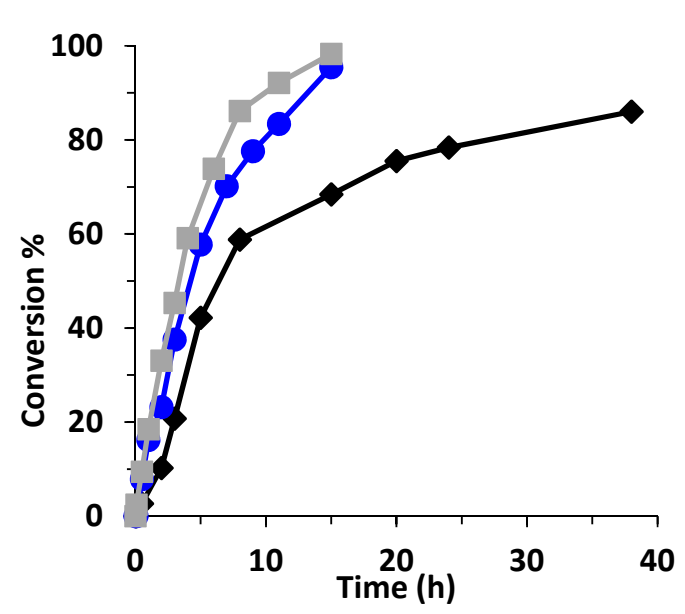

(c)

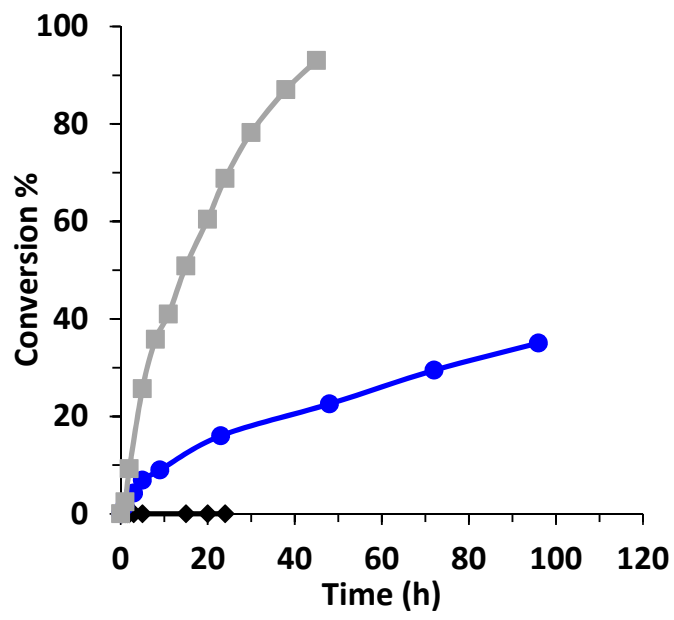

Figure 8. Conversion curves (Conversion \% vs Reaction Time (h)) for the three families of hybrid

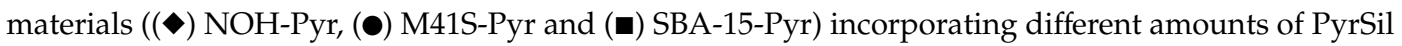
monomers: (a) $5 \mathrm{~mol} \%$, (b) $10 \mathrm{~mol} \%$, and (c) $30 \mathrm{~mol} \%$.

For hybrid materials obtained in the presence of structure-directing agents, surfactants, and block copolymers with SBA-15 and M41S topologies from the nitrogen adsorption isotherms, it was observed that the specific surface area and the pore volume decreased markedly when the amount of pyrrolidine groups inserted in the framework increased. Then, in both cases, the materials with the 
highest content of silyl-pyrrolidine fragments exhibited the lowest BET surface area. Moreover, it was notable that the solids obtained with 5 or $10 \mathrm{~mol} \%$ of PyrSil monomers exhibited similar BET area, and lower than it could be expected, probably due to the incomplete removal of surfactant and block copolymer molecules that partially blocked the pores and accessibility to active chiral centers. SBA-15and M41S-type materials with $30 \mathrm{~mol} \%$ of PyrSil fragments showed BET surface areas higher than non-ordered material, probably due to the better-achieved organization because of the presence of structure-directing agents. The catalytic performance of the SBA-15- and M41S-type materials was in agreement with the adsorption results. Indeed, the initial rate and the yield decreased when the BET surface of the materials decreased, i.e., when the accessibility to the active centers was reduced. With these families of materials, the best catalytic performances were obtained in the presence of M41S-Pyr-5\% and SBA-15-Pyr-10\% with $94 \%$ and $99 \%$ yield, respectively, and a 76\% ee (Table 3, entries 1 and 5). It is important to notice that ee was lower than in the presence of $\mathrm{NOH}-\mathrm{Pyr}-5 \%$ used as a catalyst (Table 3 , entry 1 ).

These results show that the void shapes and sizes present in the structure of hybrid materials controlled the diffusion of reactants and products, as well as confined the transition states and reactive intermediates. Therefore, the differences in initial reaction rates and enantioselectivity exhibited by the different asymmetric materials was reflective of the differences in the geometry and accessibility to the chiral active center. Likewise, the general trend of the catalytic results obtained in the presence of the different types of mesoporous materials is illustrated in Figure 9 where turnover frequency (TOF) calculated at $0.5 \mathrm{~h}$ was plotted versus BET area. It is noticeable that the catalytic activity of the synthesized hybrid materials was dependent on the BET area and the accessibility to the active centers, as expected. A maximum was observed in the presence of $\mathrm{NOH}-\mathrm{Pyr}-5 \%$, which exhibited a $636 \mathrm{~m}^{2} / \mathrm{g}$ BET area. A similar tendency was observed for the enantioselectivity process. In Figure 9, it is also clearly shown that ee\% increased with the accessibility to active sites. In contrast, catalytic data of the homogeneous pyrrolidine precursor (PyrSil) for the Michael addition between butyraldehyde and $\beta$-nitrostyrene achieved a $78 \%$ ee after $9 \mathrm{~h}$ of reaction time with a $96 \%$ of Michael adducts yield. This result confirmed that the chiral organosilyl derivative, containing pyrrolidine moieties, was successfully incorporated to the hybrid material without loss of its asymmetric catalytic properties.

(a)

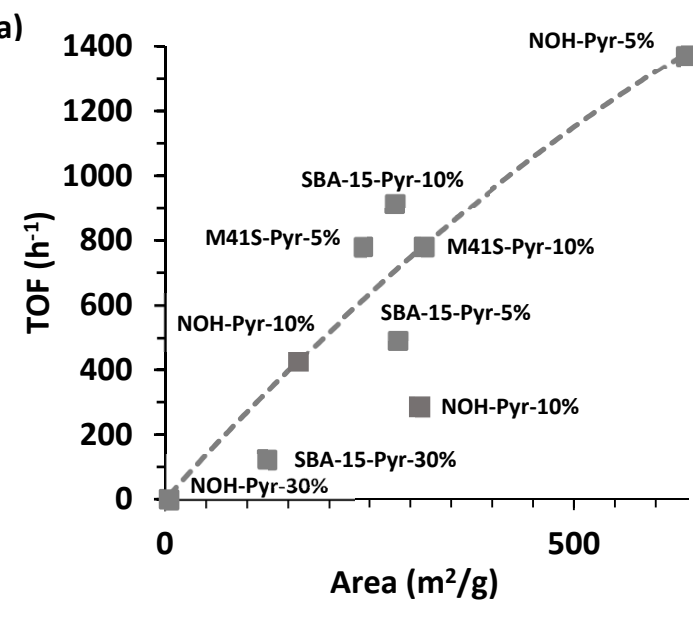

(b)

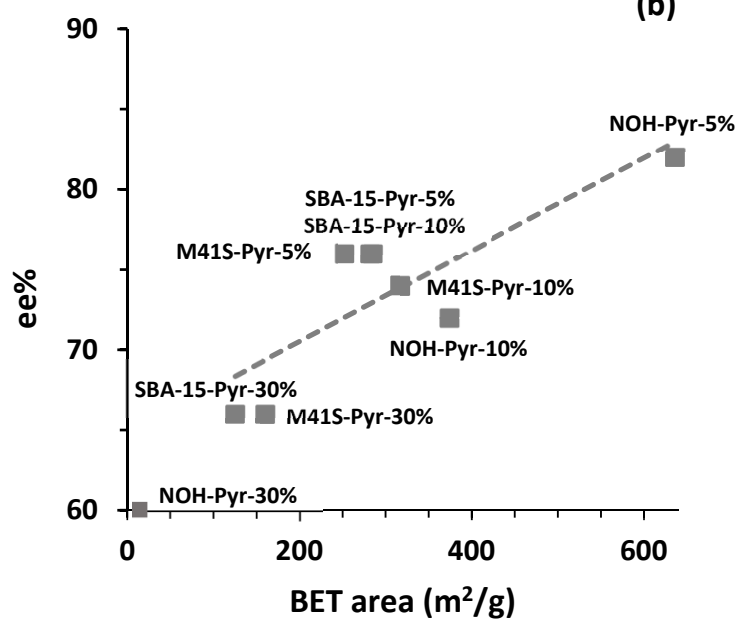

Figure 9. (a) Trend of TOF calculated at $0.5 \mathrm{~h}$ for all hybrid materials versus BET area. (b) Trend of ee\% achieved in the presence of all hybrid materials versus BET area.

Furthermore, the stability and recyclability of the different heterogeneous chiral catalysts was evaluated for four consecutive recycles (Figure 10 and Table S2). The reactions were quenched using filtration and the PyrSil hybrid materials were washed with solvent and dried before reuses. The catalytic results in terms of yield and ee for the asymmetric Michael addition of butyraldehyde to 
$\beta$-nitrostyrene showed a slight and constant decrease of the yield with increased uses ( $2-3 \%$ by run). This slow loss of yield was attributed to the strong adsorption of reactant and products that could not be completely removed by washing the catalyst after each run as it can be observed from the ${ }^{13} \mathrm{C} N \mathrm{NR}$ spectra (Figure S7) or by elemental analysis (Table S3). However, the enantio- and diasteroselectivity of each materials were fully preserved. In contrast, ${ }^{13} \mathrm{C}$ NMR spectra showed the hybrid material's structure was maintained over the uses, evidencing the robustness of the prepared catalysts.

By considering the catalytic performance of the different families of PyrSil materials and structural properties, $\mathrm{NOH}-\mathrm{Pyr}-5 \%$ hybrid material was definitively the best hybrid catalyst to perform asymmetric Michael addition with a good ee.
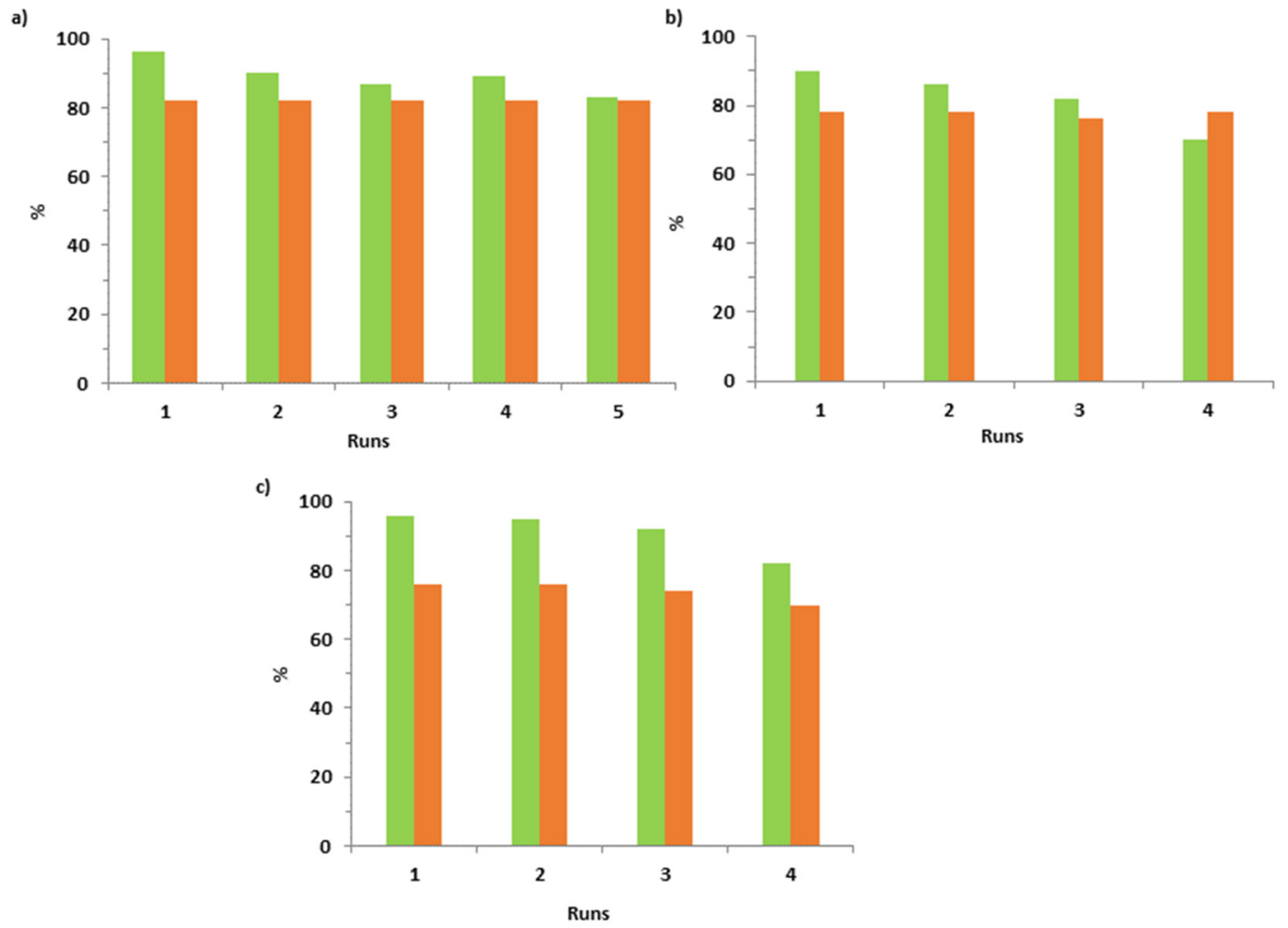

Figure 10. Catalytic performance with increased reuse of the different heterogeneous chiral hybrid catalysts for the Michael addition of butyraldehyde to $\beta$-nitrostyrene: (a) NOH-Pyr-5\%, (b) M41S-Pyr-5\%, and (c) SBA-15-Pyr-10\%. Green: (2R,3S)-2-methyl-4-nitro-3-phenylbutanal yield; Orange: ee\%.

\section{Materials and Methods}

\subsection{Synthesis of Hybrid Mesoporous Materials}

\subsubsection{Bis-Silylated Precursor PyrSil}

The organosilane precursor, (3R,5S)-5-(8,8-diethoxy-3-oxo-2,9-dioxa-4-aza-8-silaundecyl)pyrrolidin -3-yl(3-(triethoxysilyl)propyl)carbamate (PyrSil) was synthesized from (2S,4R)-4-hydroxypyrrolidine2-carboxilate hydrochloride in multistep reactions according to a reported procedure (see Scheme 1) [33]. This precursor was used to synthesize mesoporous non-ordered (NOH-Pyr) and ordered (M41S-Pyr and SBA15-Pyr) hybrid materials. 
3.1.2. Preparation of M41S-Pyr Hybrid Materials Using Surfactant Neutral Amines as Structure-Directing Agents

Mesoporous M41S-Pyr hybrid materials were synthesized via the co-condensation of bis-silylated precursor PyrSyl and TMOS with hexadecylamine as a templating agent, together with $\mathrm{PrOH}, \mathrm{EtOH}$, and water [13]. Two solutions were prepared: solution 1 was composed of TMOS and the PyrSil compound solved with $\mathrm{PrOH}$ and $\mathrm{EtOH}$, being stirred at room temperature until homogeneity, while solution 2 was composed of hexadecylamine solved with water at $40^{\circ} \mathrm{C}$ for $3 \mathrm{~h}$. Then, solution 1 was added to solution 2 under vigorous stirring, being maintained over $24 \mathrm{~h}$. The resultant mixture was aged at room temperature for $24 \mathrm{~h}$. The molar ratio achieved in the synthesis gel was 1 [(1-x)TMOS + xPyrSil] $\mathrm{SiO}_{2}$ : 0.25 hexadecylamine: $2.05 \mathrm{iPrOH}: 6.85 \mathrm{EtOH}: 66.85 \mathrm{H}_{2} \mathrm{O}\left(\mathrm{x} \mathrm{SiO}_{2} \mathrm{~mol} \%\right.$ with respect to the $\mathrm{SiO} 2$ total mol). The formed gel was aged for $24 \mathrm{~h}$ at room temperature, filtrated and dried overnight at $60{ }^{\circ} \mathrm{C}$. A fine white powder was obtained. The extraction of the surfactant from the as-synthesized solids was achieved with ethanol through four ultrasound cycles with subsequent filtration, employing $10 \mathrm{~min}$ for each cycle.

\subsubsection{Preparation of SBA-15-Pyr Hybrid Materials Using Block Copolymers as Structure-Directing} Agent

Mesoporous SBA-15-Pyr hybrid materials were synthesized via the co-condensation of chiral bis-silylated PyrSil and TMOS together with block copolymer P123 as templating agent [34]. P123, $\mathrm{NaCl}$, and $\mathrm{NH}_{4} \mathrm{~F}$ solution $(0.25 \mathrm{M})$ were mixed with $\mathrm{HCl}$ solution $(0.1 \mathrm{M})$ and water to generate a homogeneous solution that was stirred at $40^{\circ} \mathrm{C}$ for $3 \mathrm{~h}$. Then, a mixture of TMOS, PyrSil bis-silylated precursor, and ethanol was added to the solution under vigorous stirring. The resultant mixture was further stirred at $40{ }^{\circ} \mathrm{C}$ for $24 \mathrm{~h}$ and then aged at $80^{\circ} \mathrm{C}$ for $24 \mathrm{~h}$. The molar ratio achieved in the synthesis gel was $1[(1-\mathrm{x}) \mathrm{TMOS}+\mathrm{xPyrSyl}] \mathrm{SiO}_{2}: 0.017 \mathrm{P} 123: 3.42 \mathrm{NaCl}: 0.0075 \mathrm{NH}_{4} \mathrm{~F}: 166 \mathrm{H}_{2} \mathrm{O}$ : $0.03 \mathrm{HCl}: 8.7 \mathrm{EtOH}\left(\mathrm{x} \mathrm{SiO}_{2} \mathrm{~mol} \%\right.$ with respect to $\mathrm{SiO}_{2}$ total mol). As-synthesized materials were extracted with ethanol under refluxing conditions for $24 \mathrm{~h}$ to remove the structure directing agents. After filtration, resultant solids were dried at $100^{\circ} \mathrm{C}$ overnight.

3.1.4. Preparation of Mesoporous NOH-Pyr Hybrid Materials in the Absence of Structure-Directing Agents

Non-ordered hybrid mesoporous organic-inorganic materials, NOH-Pyr, were prepared from an initial mixture of tetramethoxysilane (TMOS) as silica precursor and a suitable amount of bis-silylated precursor PyrSil $\left(\left(\mathrm{R}^{\prime} \mathrm{O}\right)_{3} \mathrm{Si}-\mathrm{R}-\mathrm{Si}\left(\mathrm{OR}^{\prime}\right)_{3}\right)$ as bridged disilane in methanol [35]. After dissolution of precursors, a water solution of $\mathrm{NH}_{4} \mathrm{~F}$ was incorporated dropwise to the solution containing organosilicon alkoxide compound under vigorous stirring. The reaction mixture presented the next molar composition: 1[(1-x)TMOS + xPyrSyl]: $4 \mathrm{MeOH}: 4 \mathrm{H}_{2} \mathrm{O}: 0.00313 \mathrm{NH}_{4} \mathrm{~F}\left(x \mathrm{SiO}_{2}\right.$ mol\% with respect to $\mathrm{SiO}_{2}$ total mol) where the Si/ $\mathrm{NH}_{4} \mathrm{~F}$ and $\mathrm{TMOS} /$ disilane ratios were 479 and 4 , respectively. Hydrolysis and condensation of the silicon species were performed under vigorous and continuous stirring in a glass beaker at room temperature. Agitation was maintained until gelation. The gel was aged for $24 \mathrm{~h}$ at $36{ }^{\circ} \mathrm{C}$ and, finally, dried at $150{ }^{\circ} \mathrm{C}$ for another $24 \mathrm{~h}$. The obtained fine brown powder was profusely washed with ethanol and water in successive steps to remove the siloxane molecules that were not incorporated in the solids. Finally, solids were dried at $60^{\circ} \mathrm{C}$ overnight.

\subsection{Asymmetric Michael Additions: General Procedure}

The reaction was performed in a $3 \mathrm{~mL}$ sealed vial under magnetic stirring. Nitroalkene $(0.1 \mathrm{mmol})$, $10 \%$ of 4 -nitophenol $(0.01 \mathrm{mmol})$ and $20 \%$ of hybrid organosiliceous catalyst $(0.02 \mathrm{mmol})$ were incorporated into an aldehyde solution $(1.0 \mathrm{mmol})$ in dry DCM $(1 \mathrm{~mL})$. The resulting mixture was stirred at $15{ }^{\circ} \mathrm{C}$ for $20 \mathrm{~h}$. Then, the catalyst was recovered by filtration and washed several times with DCM and $\mathrm{H}_{2} \mathrm{O}$. The catalyst was dried in an oven at $100{ }^{\circ} \mathrm{C}$ overnight. The organic products were concentrated under reduced pressure. The Michael adducts were generated as an evaporation residue 
and purified using silica gel on Thin Layer Chromatography (TLC) plates with hexane: EtOAc $=3: 1$ as an eluent phase. Spectroscopic data of all produced products were in concordance with the previously published data $[36,37]$.

The reaction was controlled using Gas Chromatography (GC) analysis (Shimadzu, GC Plus Ultra 2010). The data of yield, selectivity, and conversion with respect to the limiting reagent (nitrostyrene derivative) were obtained from GC analysis (GC-2010-Ultra, Shimadzu equipped with an Flame Ionization Detector (FID)). Characterization of the Michael adducts was carried out using Gas Chromatography-Mass Spectrometry (GC-MS) and ${ }^{1} \mathrm{H}$ and ${ }^{13} \mathrm{C}$ NMR. Enantiomeric excess (ee), diastereoisomeric (dr), and enantiomeric ratio (er) were obtained using High-performance Liquid Chromatography (HPLC) on the purified reaction mixture using a chiral stationary phase (Chiralpak IC column).

\subsection{Characterization Techniques}

All reagents were purchased from commercial suppliers and used without further purification. CHN content was determined using a Carlo Erba 1106 elemental analyzer. Thermogravimetric and differential thermal analysis (TGA-DTA) were performed in an air stream with a Metler Toledo TGA/SDTA 851E analyzer. Nitrogen adsorption isotherms were obtained at $77 \mathrm{~K}$ with a Micromeritics ASAP 2010 volumetric adsorption apparatus. Before the analysis, the solids were outgassed for $12 \mathrm{~h}$ at $80^{\circ} \mathrm{C}$. The BET specific surface area was determined from the nitrogen adsorption data using the relative pressure range from 0.04 to 0.2 . The total pore volume was evaluated from the amount of $\mathrm{N}_{2}$ adsorbed at relative pressures around 0.99. External surface area and micropore volume were determined using the $t$-plot method in the $t$ range 3.5-5. The pore diameter and the pore size distribution were obtained using the Barret-Joyner-Halenda (BJH) methodology using the adsorption branches of the isotherms. Solid state MAS-NMR spectra were obtained at room temperature under magic angle spinning (MAS) in a Bruker AV-400 spectrometer. IR spectra of the pure precursor monomers were obtained using $\mathrm{KBr}$ disks at room temperature. Solvents used in the catalytic processes were purified by employing a solvent purification system (SPS) MBraun 800. Organic solutions were concentrated at reduced pressure using a Büchi rotary evaporator. Reactions were controlled using GC-FID (Shimadzu, GC Plus Ultra 2010) and GC-MS (Shimadzu, GCMS-QP2010 Ultra). ${ }^{1} \mathrm{H}$ and ${ }^{13} \mathrm{C}$ NMR were obtained with a Bruker 300 spectrometer and chemical shifts are given in ppm relative to residual proton solvents signals. High performance liquid chromatography (HPLC) was carried out with an Agilent Technologies 1220 Infinity Series apparatus with a Daicel Chiralpak IC $(4.6 \times 250 \mathrm{~mm})$.

\section{Conclusions}

Three families of organosiliceous materials with different structuration level, order, and textural properties (non-ordered, M41S-, and SBA15-type materials) were prepared by embedding chiral pyrrolidine units in their structural framework with various concentrations. Likewise non-ordered mesoporous hybrid solids were obtained through a sol-gel process in a fluoride medium while M41Sand SBA-15-type materials were obtained through a micellar process in the presence of long-chain neutral amine surfactants or block copolymers. The results of appropriate characterization techniques alongside catalytic study showed how the void shapes and sizes present in the structure of hybrid materials controlled the diffusion of reactants and products, as well as confined the transition states and reactive intermediates. SBA-15- and M41S-type hybrid materials with 5 or $10 \mathrm{~mol} \%$ of PyrSil exhibited similar BET areas, and lower than it was expected due to the incomplete removal of the surfactant and block copolymer molecules. Meanwhile, materials prepared with a $30 \mathrm{~mol} \%$ of PyrSil showed BET surfaces higher than non-ordered material due to the better-achieved organization in the presence of structure directing agents. Then, the best results in terms of activity and enantioselectivity were achieved in the presence of non-ordered material NOH-Pyr-5\%, which exhibited the highest BET area with a $96 \%$ yield and an $82 \%$ ee for the Michael addition between butyraldehyde and $\beta$-nitrostyrene. In this work, we showed how the differences in initial reaction rates and enantioselectivity exhibited 
by the different asymmetric materials was reflective of the differences in the geometry and accessibility to the chiral active center included in the porous hybrid framework with different topologies.

Supplementary Materials: The following are available online at http://www.mdpi.com/2073-4344/9/8/654/s1. Figure S1. Thermogravimetrical curves (TGA) and corresponding derivatives (DTA) of NOH-Pyr, as-synthesized M41S-Pyr, extracted M41S-Pyr materials, as-synthesized SBA15-Pyr, and extracted SBA15-Pyr materials with different content of bis-silylated pyrrolidine fragments. Figure S2. ${ }^{13} \mathrm{C}$ MAS NMR spectra of NOH-Pyr, M41S-Pyr, and SBA-15-Pyr materials containing different content of bis-silylated pyrrolidine fragments, obtained after extraction processes. Figure S3. ${ }^{29} \mathrm{Si} \mathrm{CP} / \mathrm{MAS}$ NMR spectra of NOH-Pyr, M41S-Pyr, and SBA-15-Pyr materials, containing different content of bis-silylated pyrrolidine fragments, obtained after extraction processes, with the assignment of T- and Q-type silicon atoms. Figure S4. ${ }^{29} \mathrm{Si}$ BD/MAS NMR spectra of NOH-Pyr, M41S-Pyr, and SBA-15-Pyr materials, containing different contents of bis-silylated pyrrolidine fragments, obtained after extraction processes, with the assignment of T- and Q-type silicon atoms. Figure S5. FTIR spectra of as-synthesized M41S-30\% and SBA-15-Pyr-30\% hybrid materials. Figure S6. $\mathrm{N}_{2}$ adsorption isotherms of NOH-Pyr, M41S-Pyr, and SBA-15-Pyr materials, containing different content of bis-silylated pyrrolidine fragments, obtained after extraction processes. Figure S7. ${ }^{13} \mathrm{C}$ NMR spectra of the different hybrid materials after four and five uses. Table S1. Integration of T-type and Q-type silicon atoms into the different catalysts obtained after extraction processes. Table S2. Catalytic results achieved in the presence of the different hybrid materials during several reaction cycles for the enantioselective Michael addition. Table S3. Elemental analysis and C/N molar ratios for NOH-Pyr, M41S-Pyr, and SBA-15-Pyr hybrid materials after extraction processes and each run. HPLC data and ${ }^{1} \mathrm{H}$ and ${ }^{13} \mathrm{C}$ NMR spectra of the Michael adduct.

Author Contributions: S.L. performed the experiments and wrote the paper; A.V. and U.D. checked the characterization results and discussion; U.D. designed the experiments, wrote the paper, and modified the article.

Funding: This research was funded by the Spanish Government (MAT2017-82288-C2-1-P), Severo Ochoa Excellence Program (SEV-2016-0683), and MULTY2HYCAT (EU-Horizon 2020 funded project under grant agreement no. 720783). S. Ll. is thankful for the predoctoral fellowship from MINECO for financial support (BES-2015-072627).

Conflicts of Interest: The authors declare no conflict of interest.

\section{References}

1. Ferey, G. Hybrid porous solids: Past, present, future. Chem. Soc. Rev. 2008, 37, 191-214. [CrossRef] [PubMed]

2. Corma, A.; Garcia, H.; Llabres i Xamena, F.X.L.I. Engineering Metal Organic Frameworks for Heterogeneous Catalysis. Chem. Rev. 2010, 110, 4606-4655. [CrossRef] [PubMed]

3. Sanchez, C.; Ribot, F. Design of hybrid organic-inorganic materials synthesized via sol-gel chemistry. New J. Chem. 1994, 18, 1007-1047.

4. Diaz, U.; Corma, A. Ordered covalent organic frameworks, COFs and PAFs. From preparation to application. Coord. Chem. Rev. 2016, 311, 85-124. [CrossRef]

5. Rowsell, J.L.C.; Yaghi, O.M. Metal-organic frameworks: A new class of porous materials. Microporous Mesoporous Mater. 2004, 73, 3-14. [CrossRef]

6. Schlichte, K.; Kratzke, T.; Kaskel, S. Improved synthesis, thermal stability and catalytic properties of the metal-organic framework compound CU3(BTC) (2). Microporous Mesoporous Mater. 2004, 73, 81-88. [CrossRef]

7. Kuschel, A.; Drescher, M.; Kuschel, T.; Polarz, S. Bifunctional Mesoporous Organosilica Materials and Their Application in Catalysis: Cooperative Effects or Not? Chem. Mater. 2010, 22, 1472-1482. [CrossRef]

8. Diaz, U.; Brunel, D.; Corma, A. Catalysis using multifunctional organosiliceous hybrid materials. Chem. Soc. Rev. 2013, 42, 4083-4097. [CrossRef] [PubMed]

9. El Kadib, A.; Molvinger, K.; Guimon, C.; Quignard, F.; Brunel, D. Design of stable nanoporous hybrid chitosan/titania as cooperative bifunctional catalysts. Chem. Mater. 2008, 20, 2198-2204. [CrossRef]

10. Horcajada, P.; Serre, C.; Vallet-Regi, M.; Sebban, M.; Taulelle, F.; Ferey, G. Metal-organic frameworks as efficient materials for drug delivery. Angew. Chem. Int. Ed. 2006, 45, 5974-5978. [CrossRef] [PubMed]

11. Zhang, J.; Han, X.; Wu, X.; Liu, Y.; Cui, Y. Chiral DHIP- and Pyrrolidine-Based Covalent Organic Frameworks for Asymmetric Catalysis. ACS Sustain. Chem. Eng. 2019, 7, 5065-5071. [CrossRef]

12. Loy, D.A.; Shea, K.J. Bridged polysilsesquioxanes-highly porous hybrid organic-inorganic materials. Chem. Rev. 1995, 95, 1431-1442. [CrossRef] 
13. Inagaki, S.; Guan, S.; Fukushima, Y.; Ohsuna, T.; Terasaki, O. Novel mesoporous materials with a uniform distribution of organic groups and inorganic oxide in their frameworks. J. Am. Chem. Soc. 1999, 121, 9611-9614. [CrossRef]

14. Villaverde, G.; Arnanz, A.; Iglesias, M.; Monge, A.; Sanchez, F.; Snejko, N. Development of homogeneous and heterogenized rhodium(I) and palladium(II) complexes with ligands based on a chiral proton sponge building block and their application as catalysts. Dalton Trans. 2011, 40, 9589-9600. [CrossRef] [PubMed]

15. Melde, B.J.; Holland, B.T.; Blanford, C.F.; Stein, A. Mesoporous sieves with unified hybrid inorganic/organic frameworks. Chem. Mater. 1999, 11, 3302-3308. [CrossRef]

16. Hoffmann, F.; Cornelius, M.; Morell, J.; Froeba, M. Silica-based mesoporous organic-inorganic hybrid materials. Angew. Chem. Int. Ed. 2006, 45, 3216-3251. [CrossRef] [PubMed]

17. Sanchez, C.; Julian, B.; Belleville, P.; Popall, M. Applications of hybrid organic-inorganic nanocomposites. J. Mater. Chem. 2005, 15, 3559-3592. [CrossRef]

18. Corma, A.; Diaz, U.; Garcia, T.; Sastre, G.; Velty, A. Multifunctional Hybrid Organic-Inorganic Catalytic Materials with a Hierarchical System of Well-Defined Micro- and Mesopores. J. Am. Chem. Soc. 2010, 132, 15011-15021. [CrossRef] [PubMed]

19. Alauzun, J.; Mehdi, A.; Reye, C.; Corriu, R.J.P. Mesoporous materials with an acidic framework and basic pores. A successful cohabitation. J. Am. Chem. Soc. 2006, 128, 8718-8719. [CrossRef]

20. Gaona, A.; Diaz, U.; Corma, A. Functional Acid and Base Hybrid Catalysts Organized by Associated (Organo)aluminosilicate Layers for C-C Bond Forming Reactions and Tandem Processes. Chem. Mater. 2017, 29, 1599-1612. [CrossRef]

21. Jones, C.W.; Tsuji, K.; Davis, M.E. Organic-functionalized molecular sieves as shape-selective catalysts. Nature 1998, 393, 52-54. [CrossRef]

22. Boronat, M.; Climent, M.J.; Corma, A.; Iborra, S.; Monton, R.; Sabater, M.J. Bifunctional Acid-Base Ionic Liquid Organocatalysts with a Controlled Distance Between Acid and Base Sites. Chem. A Eur. J. 2010, 16, 1221-1231. [CrossRef] [PubMed]

23. Garcia-Garcia, P.; Zagdoun, A.; Coperet, C.; Lesage, A.; Diaz, U.; Corma, A. In situ preparation of a multifunctional chiral hybrid organic-inorganic catalyst for asymmetric multicomponent reactions. Chem. Sci. 2013, 4, 2006-2012. [CrossRef]

24. Bass, J.D.; Anderson, S.L.; Katz, A. The effect of outer-sphere acidity on chemical reactivity in a synthetic heterogeneous base catalyst. Angew. Chem. Int. Ed. 2003, 42, 5219-5222. [CrossRef] [PubMed]

25. Kapoor, M.P.; Inagaki, S.; Ikeda, S.; Kakiuchi, K.; Suda, M.; Shimada, T. An alternate route for the synthesis of hybrid mesoporous organosilica with crystal-like pore walls from allylorganosilane precursors. J. Am. Chem. Soc. 2005, 127, 8174-8178. [CrossRef] [PubMed]

26. Garcia-Garcia, P.; Maria Moreno, J.; Diaz, U.; Bruix, M.; Corma, A. Organic-inorganic supramolecular solid catalyst boosts organic reactions in water. Nat. Commun. 2016, 7, 10835. [CrossRef] [PubMed]

27. Maria Moreno, J.; Velty, A.; Diaz, U.; Corma, A. Synthesis of 2D and 3D MOFs with tuneable Lewis acidity from preformed 1D hybrid sub-domains. Chem. Sci. 2019, 10, 2053-2066. [CrossRef] [PubMed]

28. Szollosi, G.; Gombköto, D.; Mogyorós, A.Z.; Fülöp, F. Surface-Improved Asymmetric Michael Addition Catalyzed by Amino Acids Adsorbed on Laponite. Adv. Synth. Catal. 2018, 360, 1992-2004. [CrossRef]

29. Feng, J.; Li, X.; Cheng, J.P. Enantioselective Organocatalyzed Vinylogous Michael Reactions of 3-Alkylidene Oxindoles with Enals. J. Org. Chem. 2017, 82, 1412-1419. [CrossRef]

30. Bernardi, L.; Fochi, M.; Carbone, R.; Martinelli, A.; Fox, M.E.; Cobley, C.J.; Kandagatla, B.; Oruganti, S.; Dahanukar, V.H.; Carlone, A. Organocatalytic asymmetric conjugate additions to cyclopent-1-enecarbaldehyde: A critical assessment of organocatalytic approaches towards the telaprevir bicyclic core. Chem. A Eur. J. 2015, 21, 19208-19222. [CrossRef]

31. Afewerki, S.; Ma, G.; Ibrahem, I.; Liu, L.; Sun, J.; Cordova, A. Highly Enantioselective Control of Dynamic Cascade Transformations by Dual Catalysis: Asymmetric Synthesis of Polysubstituted Spirocyclic Oxindoles. ACS Catal. 2015, 5, 1266-1272. [CrossRef]

32. Monge-Marcet, A.; Pleixats, R.; Cattoën, X.; Wong Chi Man, M.; Alonso, D.A.; Nájera, C. Prolinamide bridged silsesquioxane as an efficient, eco-compatible and recyclable chiral organocatalyst. New J. Chem. 2011, 35, 2766-2772. [CrossRef] 
33. Llopis, S.; Garcia, T.; Cantin, A.; Velty, A.; Diaz, U.; Corma, A. Chiral hybrid materials based on pyrrolidine building units to perform asymmetric Michael additions with high stereocontrol. Catal. Sci. Technol. 2018, 8, 5835-5847. [CrossRef] [PubMed]

34. Wang, P.; Liu, X.; Yang, J.; Yang, Y.; Zhang, L.; Yang, Q.; Li, C. Chirally functionalized mesoporous organosilicas with built-in BINAP ligand for asymmetric catalysis. J. Mater. Chem. 2009, 19, 8009-8014. [CrossRef]

35. Diaz, U.; Garcia, T.; Velty, A.; Corma, A. Hybrid organic-inorganic catalytic porous materials synthesized at neutral $\mathrm{pH}$ in absence of structural directing agents. J. Mater. Chem. 2009, 19, 5970-5979. [CrossRef]

36. Sagamanova, I.; Rodríguez-Escrich, C.; Molnár, I.G.; Sayalero, S.; Gilmour, R.; Pericàs, M.A. Translating the Enantioselective Michael Reaction to a Continuous Flow Paradigm with an Immobilized, Fluorinated Organocatalyst. ACS Catal. 2015, 5, 6241-6248. [CrossRef]

37. Betancort, J.M.; Barbas, C.F. Catalytic Direct Asymmetric Michael Reactions: Taming Naked Aldehyde Donors. Org. Lett. 2001, 3, 3737-3740. [CrossRef]

(C) 2019 by the authors. Licensee MDPI, Basel, Switzerland. This article is an open access article distributed under the terms and conditions of the Creative Commons Attribution (CC BY) license (http://creativecommons.org/licenses/by/4.0/). 